\title{
The Hirsch Conjecture for the fractional stable set polytope
}

\section{Journal Article}

\section{Author(s):}

Michini, Carla; Sassano, Antonio

Publication date:

2014-10

Permanent link:

https://doi.org/10.3929/ethz-b-000074180

Rights / license:

In Copyright - Non-Commercial Use Permitted

Originally published in:

Mathematical Programming 147(1-2), https://doi.org/10.1007/s10107-013-0723-3 


\title{
The Hirsch Conjecture for the fractional stable set polytope
}

\author{
Carla Michini · Antonio Sassano
}

Received: 3 June 2012 / Accepted: 16 October 2013 / Published online: 29 November 2013

(C) Springer-Verlag Berlin Heidelberg and Mathematical Optimization Society 2013

\begin{abstract}
The edge formulation of the stable set problem is defined by two-variable constraints, one for each edge of a graph $G$, expressing the simple condition that two adjacent nodes cannot belong to a stable set. We study the fractional stable set polytope, i.e. the polytope defined by the linear relaxation of the edge formulation. Even if this polytope is a weak approximation of the stable set polytope, its simple geometrical structure provides deep theoretical insight as well as interesting algorithmic opportunities. Exploiting a graphic characterization of the bases, we first redefine pivots in terms of simple graphic operations, that turn a given basis into an adjacent one. These results lead us to prove that the combinatorial diameter of the fractional stable set polytope is at most the number of nodes of the given graph. As a corollary, the Hirsch bound holds for this class of polytopes.
\end{abstract}

Keywords Polytope $\cdot$ Diameter $\cdot$ Stable set problem

Mathematics Subject Classification $\quad 90 \mathrm{C} 08 \cdot 90 \mathrm{C} 27 \cdot 90 \mathrm{C} 35 \cdot 90 \mathrm{C} 49 \cdot$ 90C57

\section{Introduction}

The combinatorial diameter of a polyhedron is the smallest natural number that bounds the length of a shortest path connecting two arbitrary vertices (i.e. 0-dimensional faces) of the polyhedron along its edges (i.e. 1-dimesional faces). The Hirsch Conjecture (1957) states that a $d$-dimensional polyhedron with $f$ facets cannot have combinator-

C. Michini $(\bowtie)$

ETH Zürich, Zürich, Switzerland

e-mail: carla.michini@ifor.math.ethz.ch

A. Sassano

Sapienza Università di Roma, Rome, Italy

e-mail: sassano@dis.uniroma1.it 
ial diameter greater than $f-d$. The importance of the Hirsch Conjecture is related to its connection with the performance of the simplex method for linear programming, because the diameter of a polyhedron is a lower bound on the number of pivots needed by the algorithm to reach an optimal vertex from an arbitrary one. The Hirsch Conjecture has been disproved first for unbounded polyhedra [10], and recently also for bounded ones [15]. Although we know that it does not hold in general, it is still interesting to investigate families of polyhedra that satisfy the Hirsch bound. For example, it has been proven by Naddef [12] that the diameter of a $d$-dimensional $(0,1)$-polytope is at most $d$, and that $(0,1)$-polytopes satisfy the Hirsch bound. The diameter of various polyhedra arising from network problems has also been object of research. In many cases the special structure of these polyhedra allows to establish specific characterizations of the vertices, and easier conditions for their adjacency. Such properties can be exploited to bound the diameter of these polyhedra. Examples of network polyhedra satisfying the Hirsch bound are the assignment polytope [3], signature polytopes [4] and the dual of the transportation polyhedron [2]. Serious efforts have also been made to tighten the bounds on the diameter of the transportation polytope [5]. The present paper is an example of this approach. In this work we study the fractional stable set polytope $\operatorname{FSTAB}(G)$, i.e. the polytope defined by the linear relaxation of the edge formulation for the stable set problem on graph $G$. Unlike the polyhedra mentioned above, the fractional stable set polytope is not integral and it is generally a weak relaxation of the stable set polytope. Even if the stable set polytope is a $(0,1)$-polytope and thus it satisfies the Hirsch bound, the number of its facets is exponential and, in practice, the linear program given by its facet-description is intractable. This motivates our interest in the fractional stable set polytope, that has reasonably many facets, but is not a $(0,1)$-polytope anymore, and may or may not satisfy the Hirsch bound. Our main result is that the combinatorial diameter of the fractional stable set polytope is at most the cardinality of the node set of the graph. As a corollary, the Hirsch bound holds for this class of polytopes. In order to prove our result, we use a graphic characterization of the bases of the fractional stable set polytope, and we derive graphic conditions for the adjacency of two bases. Moreover, it is known that the fractional stable set polytope has the Trubin property [16], i.e. two integer vertices are adjacent on this fractional polytope if and only if they are adjacent on the stable set polytope. As a consequence, the characterization of bases and pivots that we present opens new algorithmic opportunities for the solution of the stable set problem.

We denote the node set and the edge set of a graph $G$ as $V(G)$ and $E(G)$, respectively. We call singleton or isolated node the graph consisting of a single node. Let $G=(V, E)$ and $U \subseteq V$. We denote the neighbors of $U$ by $N(U)=\{v \in V: u v \in$ $E, u \in U, v \in V \backslash U\}$. We write $G[U]$ to indicate the subgraph of $G$ induced by nodes of $U \subseteq V$. Moreover, for a vector $x \in \mathbb{R}^{|V|}$, we denote by $x_{U}$ the subvector of $x$ indexed by $U$. For a cut $(U, V \backslash U)$, we indicate the corresponding cutset as $\delta(U)=\{u v \in E: u \in U, v \in V \backslash U\}$. We denote the union of two graphs $G$ and $G^{\prime}$ as $G \cup G^{\prime}$, where $V\left(G \cup G^{\prime}\right)=V(G) \cup V\left(G^{\prime}\right)$ and $E\left(G \cup G^{\prime}\right)=E(G) \cup E\left(G^{\prime}\right)$.

The paper is organized as follows. In Sect. 2 we present a graphic characterization of bases of $\operatorname{FSTAB}(G)$; in Sect. 3 we introduce a graphic interpretation of pivots; in Sect. 4 we characterize the adjacency between fractional and integer vertices; in 
Sect. 5 we prove the bound of $|V(G)|$ for the combinatorial diameter of $\operatorname{FSTAB}(G)$, and we then show that the Hirsch bound is valid for $\operatorname{FSTAB}(G)$.

\section{The fractional stable set polytope}

Let $G=(V, E)$ be a simple, undirected graph, where $V$ and $E$ are the sets of $n$ nodes and $m$ edges of $G$, respectively, and let $c: V \rightarrow \mathbb{Q}_{+}$be any weighting of the nodes of $G$. A stable set is a subset $S$ of the node set $V$ such that no two vertices of $S$ are adjacent. The stable set problem (STAB) asks for the stable set of maximum weight and it is a well known NP-hard problem. The stable set polytope, denoted by $\operatorname{STAB}(G)$ is the convex hull of the incidence vectors of stable sets in $G$. A straightforward formulation of the stable set problem is the so-called edge formulation:

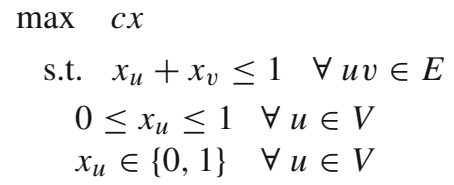

where constraints (1) express the simple condition that the endpoints of an edge cannot both belong to a stable set. The fractional stable set polytope, denoted by $\operatorname{FSTAB}(G)$, is the polytope defined by inequalities (1) and (2). We denote by (FSTAB) the linear relaxation of the edge formulation, i.e. $\max \{c x: x \in \operatorname{FSTAB}(G)\}$. It is well known that $\operatorname{FSTAB}(G)$ coincides with $\operatorname{STAB}(G)$ if and only if $G$ is bipartite. It was originally proven by Balinski that vertices of $\operatorname{FSTAB}(G)$ are $\left(0, \frac{1}{2}, 1\right)$-valued $[1,13,14]$. Moreover, variables that are integer valued in an optimal solution to (FSTAB) can be fixed to solve (STAB) [13].

Let $Z \subseteq V$ be the set of isolated nodes of $G$. Note that the constraints $x_{u} \leq 1$ are redundant for each node $u \in V \backslash Z$. It is easy to verify that the nonnegativity constraints $x_{u} \geq 0, u \in V$, and the constraints $x_{u} \leq 1, u \in Z$, are facets of $\operatorname{FSTAB}(G)$. To show that inequalities (1) are facet defining, let $u v \in E$ and for each $w \in V$ define vector $x^{w}$ as follows: for $w \notin\{u, v\}, x_{u}^{w}=x_{v}^{w}=x_{w}^{w}=\frac{1}{2}$ and $x_{z}^{w}=0 \forall z \notin\{u, v, w\}$; for $w=u, x_{u}^{u}=1$ and $x_{z}^{u}=0 \forall z \neq u$; for $w=v, x_{v}^{v}=1$ and $x_{z}^{v}=0 \forall z \neq v$. For all $w \in V, x^{w} \in \operatorname{FSTAB}(G)$ and $x_{u}^{w}+x_{v}^{w}=1$. It is not hard to check that this is a set of $|V|$ vectors that are affinely independent. This implies, since $P$ is full-dimensional, that the Hirsch bound equals $|E|+|Z|$.

Suppose that $G$ consists of $k$ connected components $G^{1}, \ldots, G^{k}$ and observe that $\operatorname{FSTAB}(G)=\operatorname{FSTAB}\left(G^{1}\right) \times \cdots \times \operatorname{FSTAB}\left(G^{k}\right)$. We denote by $d(\operatorname{FSTAB}(G))$ the diameter of $\operatorname{FSTAB}(G)$ and by $d\left(\operatorname{FSTAB}\left(G^{i}\right)\right)$ the diameter of $\operatorname{FSTAB}\left(G^{i}\right), i=$ $1, \ldots, k$. We next remark that, in order to bound the diameter of $\operatorname{FSTAB}(G)$, we can bound the diameter of each polytope $\operatorname{FSTAB}\left(G^{i}\right), i=1, \ldots, k$.

Remark 1 Let $x$ and $y$ be two vertices of $\operatorname{FSTAB}(G)$, and express them as

$$
x=\left(\begin{array}{c}
x^{1} \\
\vdots \\
x^{k}
\end{array}\right), \quad y=\left(\begin{array}{c}
y^{1} \\
\vdots \\
y^{k}
\end{array}\right) \text {, }
$$


where $x^{i}, y^{i} \in \operatorname{FSTAB}\left(G^{i}\right), i=1, \ldots, k$. Then, $x$ and $y$ are adjacent on $\operatorname{FSTAB}(G)$ if and only if $x^{j}$ and $y^{j}$ are adjacent on $\operatorname{FSTAB}\left(G^{j}\right)$ for some $j \in\{1, \ldots, k\}$ and $x^{i}=y^{i}$ for all $i \neq j$. Therefore $d(\operatorname{FSTAB}(G))=\sum_{i=1}^{k} d\left(\operatorname{FSTAB}\left(G^{i}\right)\right)$.

If $G^{i}$ is an isolated node, then $\operatorname{FSTAB}\left(G^{i}\right)=\{x \in \mathbb{R}: 0 \leq x \leq 1\}$, and $d\left(\operatorname{FSTAB}\left(G^{i}\right)\right)=1$.

Let $G^{i}$ be a connected component of $G$ that has at least an edge. If $G^{i}$ is bipartite, $\operatorname{FSTAB}\left(G^{i}\right)=\operatorname{STAB}\left(G^{i}\right)$, and $d\left(\operatorname{FSTAB}\left(G^{i}\right)\right) \leq \min \left\{\left|V\left(G^{i}\right)\right|,\left|E\left(G^{i}\right)\right|\right\}$ [12]. Note that, in this case, $\left|E\left(G^{i}\right)\right|<\left|V\left(G^{i}\right)\right|$ if and only if $G^{i}$ is a tree. If $G^{i}$ is nonbipartite, we prove that $d\left(\operatorname{FSTAB}\left(G^{i}\right)\right) \leq\left|V\left(G^{i}\right)\right|$. Note that, in this case, $\left|V\left(G^{i}\right)\right| \leq\left|E\left(G^{i}\right)\right|$.

In order to prove that the diameter of $\operatorname{FSTAB}(G)$ is bounded by $|V(G)|$ from now on, unless explicitly stated, we assume the following.

Assumption 2.1 $G=(V, E)$ is a connected graph with at least one edge.

We will show that, from an arbitrary vertex $x$ of $\operatorname{FSTAB}(G)$, it is possible to reach any other vertex $x^{\prime}$ through a sequence of adjacent vertices $\left\{x^{t}\right\}_{t=0, \ldots, T}$, such that $x^{0}=x, x^{T}=x^{\prime}$ and $T \leq|V|$. For each vertex $x^{t}$ of the sequence $\left\{x^{t}\right\}$, we define the set $V^{\prime}(t)=\left\{u \in V: x_{u}^{t}=x_{u}^{\prime}\right\}$. Our sequence will satisfy the following conditions: $V^{\prime}(t+1) \supseteq V^{\prime}(t)$ for all $t=0, \ldots, T-1$ and $V^{\prime}(T)=V$, i.e. $x^{T}=x^{\prime}$.

To prove our results, we heavily exploit a graphic characterization of the bases of $\operatorname{FSTAB}(G)$, presented in Campêlo and Cornuéjols [6], Cornuéjols et al. [7]. The graphic characterization, independently investigated by Michini in [11], is based upon a slight rewriting of $\operatorname{FSTAB}(G)$, that is obtained by converting (FSTAB) to standard form [8]: a nonnegative slack variable is introduced for each edge constraint, i.e. $\operatorname{FSTAB}(G)=\left\{(x, y) \in \mathbb{R}_{+}^{n} \times \mathbb{R}_{+}^{m}: x_{u}+x_{v}+y_{u v}=1 \quad \forall u v \in E\right\}$. Clearly, every node of $G$ corresponds to an $x$ variable of $\operatorname{FSTAB}(G)$ and each edge of $G$ corresponds to a $y$ slack variable of $\operatorname{FSTAB}(G)$. Therefore, in the rest of the paper, we will call the $x$ and $y$ variables node and edge variables, respectively. We will say that a node (resp. an edge) is $0, \frac{1}{2}$ or 1 valued if the corresponding node (resp. edge) variable is $0, \frac{1}{2}$ or 1 valued, respectively.

Let $A$ be the edge-node incidence matrix of $G$, with $m$ rows, indexed by the edges of $G$ and $n$ columns, indexed by the nodes of $G$. Denote by $\mathcal{B}$ the set of all bases of the constraint matrix $\left[\begin{array}{ll}A & I_{m}\end{array}\right]$. Since the rows of the constraint matrix defining $\operatorname{FSTAB}(G)$ are linearly independent, any basis consists of $m$ columns. We say that two bases are adjacent if they share $m-1$ columns. Moreover, we define a basis to be integral if its associated basic solution is integral. For every basis, we denote by $B$ and $N$ the corresponding basic and nonbasic submatrices, respectively. For conciseness, we will also use $B$ and $N$ to denote the associated index sets. For classical terminology on linear programming we refer to [8].

Let $B \in \mathcal{B}$ be a basis, feasible or infeasible, of $\left[\begin{array}{ll}A & I_{m}\end{array}\right]$. Partition the nodes of $G$ according to $B$ into $V_{B}$ and $V_{N}$, indexing the basic and nonbasic node variables $x_{B}$ and $x_{N}$, respectively. Similarly, partition the edges into $E_{B}$ and $E_{N}$ and the corresponding edge variables into $y_{B}$ and $y_{N}$. In order to characterize the structure of the basis, consider the basic subgraph $G_{B}=\left(V, E_{N}\right)$, which is obtained from $G$ by removing the basic edges. Let $C_{i}=\left(V_{i}, E_{i}\right), i=1, \ldots, k$, be the connected components of $G_{B}$. 
Recall that a graph is a 1-tree if it is connected and the number of its nodes equals the number of its edges. A 1-tree contains a unique cycle. We call 1-pseudoforest a subgraph composed by rooted trees and 1-trees with an odd cycle. We remark that every singleton of $G_{B}$ can be seen as a trivial tree, containing only one node and no edges.

Theorem 1 (Campêlo and Cornuéjols [6]) For every $B \in \mathcal{B}, G_{B}$ is a 1-pseudoforest of $G$. The root of each tree is the only nonbasic node of the tree. The nodes of every 1-tree are all basic.

The converse of Theorem 1 also holds [7]. This implies that there is a one-to-one correspondence between 1-pseudoforests of $G$ and bases of $\operatorname{FSTAB}(G)$.

Theorem 2 (Cornuéjols, Michini and Nannicini [7]) Let $F$ be a 1-pseudoforest of $G$ with $V(F)=V$ and denote by $R \subseteq V$ the subset of roots of $F$. Let $V_{B}=V \backslash R, E_{B}=$ $E \backslash E(F)$. Then $B=V_{B} \cup E_{B}$ is a basis of $\operatorname{FSTAB}(G)$ and $G_{B}=F$.

Theorems 1 and 2 establish a precise correspondence between bases of $\operatorname{FSTAB}(G)$ and 1-pseudoforests of $G$. Given a basis $B \in \mathcal{B}$ and the associated 1-pseudoforest $G_{B}$, define $\mathcal{I}_{0}(B)$ and $\mathcal{I}_{1}(B)$ as the subsets of $\{1, \ldots, k\}$ indexing the tree and 1tree components of $G_{B}$, respectively. For every rooted tree of $G_{B}$, i.e. for each $C_{i}$ with $i \in \mathcal{I}_{0}(B)$, we denote by $\tau\left(C_{i}\right)$ the root of the tree. Similarly, for every 1-tree component $C_{j}, j \in \mathcal{I}_{1}(B)$, of $G_{B}$, we denote by $\kappa\left(C_{j}\right)$ its unique (odd) cycle.

The next theorem highlights the connection between the variables that are $\frac{1}{2}$-valued in a basic solution of $\operatorname{FSTAB}(G)$ and the nodes belonging to the 1-tree components of the associated basic subgraph.

Theorem 3 (Cornuéjols, Michini and Nannicini [7]) Let $B \in \mathcal{B}$ be a basis of $\operatorname{FSTAB}(G)$. Denote by $\bar{x}$ the basic solution associated to $B$. Then:

(i) all nodes in 1-tree components of $G_{B}$ index $\frac{1}{2}$-valued components of $\bar{x}$;

(ii) all nodes of tree components of $G_{B}$ index $(0,1)$-valued components of $\bar{x}$. In each tree component, nodes that are at even distance from the root are 0-valued, while those that are at odd distance from the root are 1-valued.

Graphically, we will represent zero, one and half valued nodes by white, black and half colored circles, respectively. For each tree, the node corresponding to its root will be circled. Nonbasic edges will be represented by solid lines, and basic edges by dashed lines. As an example, Fig. 1 represents two degenerate bases of a fractional vertex and their corresponding basic subgraphs.

\section{A graphic characterization of pivots}

In this section we present a graphic characterization for the adjacency of two bases. The operation of moving from a basis to an adjacent basis is called a pivot [8]. The characterization of bases of $\operatorname{FSTAB}(G)$ presented in Sect. 2 allows us to graphically describe pivots as well. A similar task was tackled by Ikura and Nemhauser for bipartite graphs [9]. Given a basis $B \in \mathcal{B}$, pivots on $\operatorname{FSTAB}(G)$ can be characterized in terms 

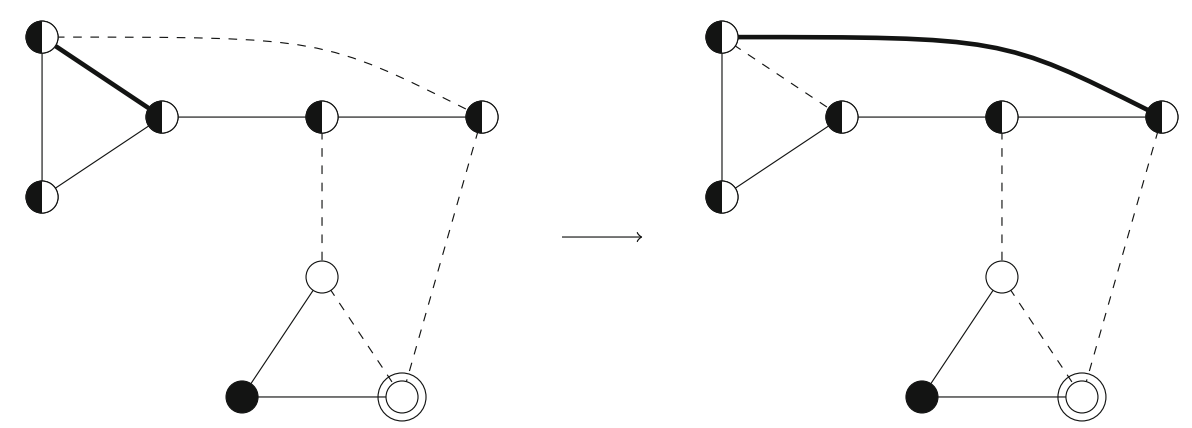

Fig. 1 EDGE-OUT + EDGE-IN operations. The pivot in the example is degenerate
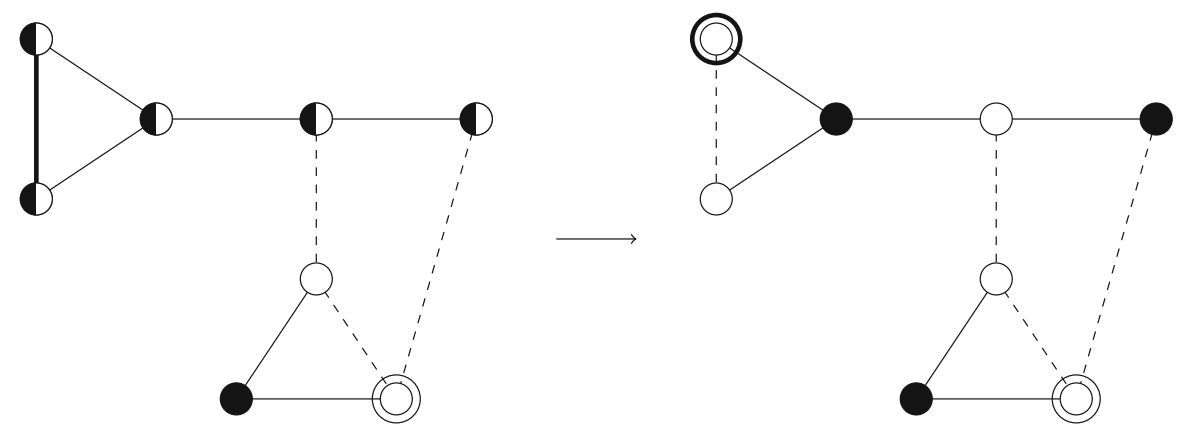

Fig. 2 EDGE-OUT + ROOT-IN operations. The pivot in the example is nondegenerate and feasible. Moreover, the pivot "pushes" integrality

of elementary transformations of $G_{B}$ into $G_{B^{\prime}}$, where $B^{\prime} \in \mathcal{B}$ is adjacent to $B$ in $\operatorname{FSTAB}(G)$. In Michini [11] an overview of all possible transformations of $G_{B}$ into $G_{B^{\prime}}$ is presented. Between all possible pivots, degenerate and nondegenerate ones are characterized, as well as those leading to an integer or to a fractional vertex.

In the following, we provide an essential and intuitive description of such results, by defining the pivots on $\operatorname{FSTAB}(G)$ in general terms.

Definition 1 Given a 1-pseudoforest $G_{B}$, we define the following OUT-operations:

(i) $E D G E-O U T$ : remove edge $u v \in E_{N}$ (i.e. the edge variable $y_{u v}$ enters the basis);

(ii) ROOT-OUT: unroot a tree component $C_{k}$ (i.e. the node variable indexed by $\tau\left(C_{k}\right) \in V_{N}$ enters the basis);

When we perform an EDGE-OUT operation on $G_{B}$, we either open an odd cycle of the 1-pseudoforest (Figs. 1 and 2), or we break one of its connected components (Fig. 3 ). In any case, the operation yields an unrooted tree. Similarly, when we perform a ROOT-OUT operation, we obtain an unrooted tree.

Definition 2 Let $G_{B}^{\prime}$ be the subgraph obtained from a 1-pseudoforest $G_{B}$ by applying a OUT-operation, and let $C_{k}^{\prime}$ be its unique unrooted tree. We define the following $I N$ operations: 


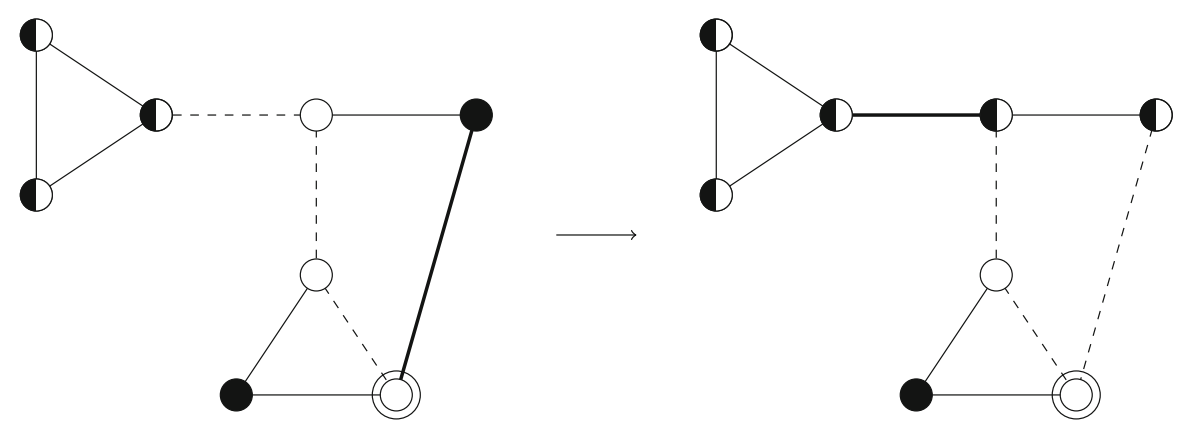

Fig. 3 EDGE-OUT + EDGE-IN operations. The pivot in the example is nondegenerate and feasible. Moreover, the pivot "relaxes" integrality

(i) $E D G E$-IN : add edge $u v \in E_{B}$, such that either $u v$ is in $\delta\left(V\left(C_{k}^{\prime}\right)\right)$ or it closes an odd cycle in $C_{k}^{\prime}$ (i.e. the edge variable $y_{u v}$ leaves the basis);

(ii) ROOT-IN: root $C_{k}^{\prime}$ at node $u \in V\left(C_{k}^{\prime}\right) \cap V_{B}$ (i.e. the node variable $x_{u}$ leaves the basis).

Because any OUT-operation yields an unrooted tree $C_{k}^{\prime}$, to obtain a new 1-pseudoforest, we can perform a ROOT-IN operation to root $C_{k}^{\prime}$ (Fig. 2). Alternatively, an EDGE-IN operation can be applied; in this case the edge that is added to the current subgraph can either close an odd cycle in $C_{k}^{\prime}$ (Fig. 1) or it can connect $C_{k}^{\prime}$ to another tree or 1-tree component of $G_{B}^{\prime}$ (Fig. 3).

Definition 3 An elementary transformation on a 1-pseudoforest $G_{B}$ is defined as a sequential application of an OUT-operation and an IN-operation.

Definition 4 Two 1-pseudoforests $G_{B}$ and $G_{B^{\prime}}$ are said to be adjacent if $B$ and $B^{\prime}$ are adjacent bases.

Theorem 4 Let $B$ and $B^{\prime}$ be two bases of $\operatorname{FSTAB}(G)$, and let $G_{B}$ and $G_{B^{\prime}}$ be the associated 1-pseudoforests. A pivot from $B$ to $B^{\prime}$ is an elementary transformation that turns $G_{B}$ into $G_{B^{\prime}}$. Moreover, any elementary transformation on $G_{B}$ is a pivot from $B$ to an adjacent basis.

Proof $B$ and $B^{\prime}$ have $m-1$ variables in common. Let $h \in B \backslash B^{\prime}$ and $k \in B^{\prime} \backslash B$. A pivot from $B$ to $B^{\prime}$ consists in the elementary transformation where an OUT-operation is performed on $k$ and an IN-operation is performed on $h$. Depending on $k$ (resp. $h$ ) being a node or an edge variable, we have a ROOT-OUT or an EDGE-OUT (resp. a ROOT-IN or an EDGE-IN) operation.

Suppose now to perform an elementary transformation on $G_{B}$. We obtain a new 1pseudoforest $F$ with $V(F)=V$. Let $R \subseteq V$ denote the subset of roots of $F$. Then, by Theorem 2, $(V \backslash R) \cup(E \backslash E(F))=B^{\prime} \in \mathcal{B}$ and $G_{B^{\prime}}=F$. Moreover, as an elementary transformation consists only of an OUT-operation and an IN-operation, it follows that $B$ and $B^{\prime}$ have $m-1$ variables in common, i.e. they are adjacent.

When we perform a pivot, the only (node) variables involved in the transformation are those belonging to the unrooted tree arising from the EDGE-OUT or ROOT-OUT 
operation. If the pivot is nondegenerate, these are the only variables whose value will change in the new basic solution (Figs. 2 and 3). Note that a pivot is degenerate if and only if, in the elementary transformation corresponding to the pivot, the variable involved in the IN-operation (i.e. the variable leaving the basis) is 0-valued (Fig. 1).

A nondegenerate pivot is feasible if it yields a new basic feasible solution, i.e. a basic solution whose (basic) edge variables are all nonnegative. Between nondegenerate pivots, there are some "pushing" and some "relaxing" integralities. Consider the pivot from $B$ to $B^{\prime}$ and let $C_{k}^{\prime}$ be the unrooted tree yielded by the OUT-operation performed on $G_{B}$. If the IN-operation is such that the nodes in $V\left(C_{k}^{\prime}\right)$ belong to a tree in $G_{B}$ and to a 1-tree in $G_{B^{\prime}}$, then the node variables associated to $V\left(C_{k}^{\prime}\right)$, that were originally integer valued, become fractional (Fig. 3). Vice versa, if the nodes of $V\left(C_{k}^{\prime}\right)$ were in a 1-tree in $G_{B}$ and become part of a tree in $G_{B^{\prime}}$, then integrality is achieved on the corresponding node variables (Fig. 2).

\section{Adjacency of integer and fractional vertices}

Theorems 1, 2 and 3 immediately imply that $\operatorname{FSTAB}(G)$ admits fractional vertices if and only if $G=(V, E)$ is nonbipartite. In this section, we characterize the adjacency between fractional and integer vertices of the fractional stable set polytope.

Two vertices $x$ and $x^{\prime}$ of $\operatorname{FSTAB}(G)$ are adjacent if there exist two adjacent bases $B, B^{\prime} \in \mathcal{B}$ associated to $x$ and $x^{\prime}$, respectively. Equivalently, $x$ and $x^{\prime}$ are adjacent if there exist two adjacent 1-pseudoforests $G_{B}$ and $G_{B^{\prime}}$ associated to $x$ and $x^{\prime}$, respectively. In [14] Padberg gave a characterization of the fractional vertices of $\operatorname{FSTAB}(G)$ that are adjacent to a given integer vertex, by proving that for each vertex of this type there exists a suitably structured basic matrix. In the next Lemma, we restate the result of Padberg in terms of our graphic characterization of bases.

Lemma 1 If $B$ is a non-integral basis adjacent to an integral basis, then $G_{B}$ contains exactly one 1-tree component.

Given a vector $x \in\left\{0, \frac{1}{2}, 1\right\}^{|V|}$, define $H(x)=\left\{u \in V: x_{u}=\frac{1}{2}\right\}$ and $I(x)=$ $\left\{u \in V: x_{u} \in\{0,1\}\right\}$.

Lemma 2 Let $x$ be a fractional vertex of $\operatorname{FSTAB}(G)$ and $x^{\prime}$ be the incidence vector of a stable set $S$ of $G$. Then $x$ and $x^{\prime}$ are adjacent on $\operatorname{FSTAB}(G)$ if and only if $x_{u}=x_{u}^{\prime} \forall u \in I(x)$ and there exists a spanning tree $T$ of $G[H(x)]$ such that for each $u v \in E(T), u v \in \delta(S)$.

Proof Suppose that $x_{u}=x_{u}^{\prime} \forall u \in I(x)$ and that there exists a spanning tree $T$ of $G[H(x)]$ with each edge connecting a node in $S$ and a node in $V \backslash S$. We will show that $x$ and $x^{\prime}$ are adjacent by defining a suitable basis of $x^{\prime}$ such that, in one pivot, we can reach a basis associated to $x$. Consider an arbitrary basis $B$ associated to $x$ and let $C_{i}=\left(V_{i}, E_{i}\right), i=1, \ldots, r$, be the trees of $G_{B}$. Note that $I(x)=\bigcup_{i=1}^{r} V_{i}$ and that if $r=0$ then $I(x)=\emptyset$. Define the rooted tree $C_{r+1}=T$, by setting $\tau\left(C_{r+1}\right)=z$, with

$z \in H(x) \backslash S$. By Theorems 2 and 3, the 1-pseudoforest $F=\bigcup_{i=1}^{r+1} C_{i}$ defines a basis $B^{\prime}$ associated to $x^{\prime}$ such that $G_{B^{\prime}}=F$. Recall that, since $x$ is a vertex of $\operatorname{FSTAB}(G)$, it 

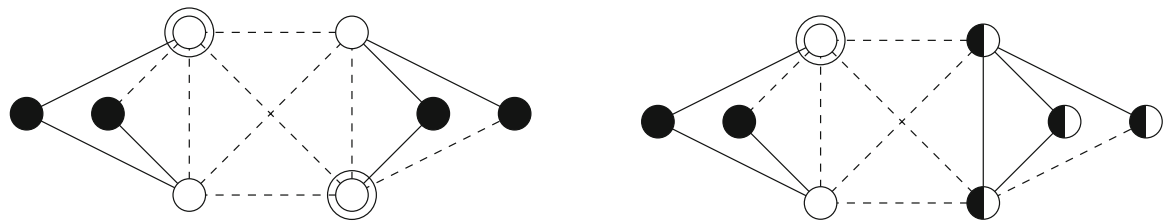

Fig. 4 An integer and a fractional adjacent vertices. Note that the integer vertex is not adjacent to the solution $x_{i}^{*}=\frac{1}{2}$ for all $i=1, \ldots, n$, because it admits no spanning tree satisfying the conditions of Lemma 2

follows that the induced subgraph $G[H(x)]$ is not bipartite. Therefore, there exists an edge $h j$ such that $h, j \in H(x) \backslash S$. Note that $x_{h}^{\prime}=x_{j}^{\prime}=0$, i.e. $h j \notin E\left(G_{B^{\prime}}\right)$. We can then perform the following elementary transformation on $G_{B^{\prime}}$ : a ROOT-OUT operation on $z$ and an EDGE-IN operation on $h j$. This yields a 1-pseudoforest $G_{\bar{B}}$ associated to basis $\bar{B}=B^{\prime} \backslash h j \cup\{z\}$. Precisely, $G_{\bar{B}}$ is composed by the trees $\bar{C}_{i}=C_{i}, i=1, \ldots, r$, and the 1-tree component $\bar{C}_{r+1}=T \cup h j$. By Theorem 3, the basic feasible solution associated to $\bar{B}$ is $x$, i.e. $\bar{B}$ is a basis associated to $x$ that is adjacent to $B^{\prime}$.

Suppose now that $x^{\prime}$ is adjacent to $x$ on $\operatorname{FSTAB}(G)$. By Lemma 1 , there exist two adjacent bases $B^{\prime}$ and $B$, associated to $x^{\prime}$ and $x$, respectively, such that $G_{B}$ contains only a 1 -tree component. Let $C_{i}, i=1, \ldots, r$, be the tree components of $G_{B}$ and let $C_{r+1}(H(x), W)$ be its unique 1-tree. By Theorem 4, the pivot leading to $B^{\prime}$ corresponds to an elementary transformation that turns $G_{B}$ into $G_{B^{\prime}}$. As the node variables in $H(x)$ are binary in $x^{\prime}$, by Theorem 3, in $G_{B^{\prime}}$ such variables belong to tree components. As a consequence, in the elementary transformation turning $G_{B}$ into $G_{B^{\prime}}$, the OUT-operation is an EDGE-OUT operation on some edge $h j \in \kappa\left(C_{r+1}\right)$. After the EDGE-OUT operation on $h j$ we obtain the unrooted tree $T=(H(x), W \backslash h j)$, implying that the only variables changing value in $x^{\prime}$ are those indexed by $H(x)$, i.e. $x_{u}=x_{u}^{\prime} \forall u \in I(x)$. In the IN-operation that, together with the EDGE-OUT operation on $h j$, defines the pivot from $B$ to $B^{\prime}, T$ will be either rooted at a node $z \in H(x) \backslash S$ through a ROOT-IN operation, becoming a rooted tree of $G_{B^{\prime}}$, or it will be merged to a rooted tree $C_{i}, i \in\{1, \ldots, r\}$ through an EDGE-IN operation on some edge of $\delta(H(x))$. Because all the edges of $G_{B^{\prime}}$ are nonbasic, and therefore 0 -valued, it follows that each edge in $T$ connects a node in $S$ and a node in $V \backslash S$.

An illustration of the condition of Lemma 2 is depicted in Fig. 4.

In the following definition we introduce minimal bases, a crucial property to preserve feasibility when we perform a ROOT-OUT plus an EDGE-IN elementary transformation that merges two trees of a 1-pseudoforest.

Definition 5 A basis $B$ is called minimal if any two tree components of $G_{B}$ are only connected by 1 -valued (basic) edges.

A basis $B \in \mathcal{B}$ is trivially minimal if $G_{B}$ has at most one tree component, i.e. if $\left|\mathcal{I}_{0}(B)\right| \leq 1$.

Lemma 3 Let $x$ be a vertex of $\operatorname{FSTAB}(G)$, and let $B$ be a basis of $x$ with $\left|\mathcal{I}_{0}(B)\right| \geq 2$. There always exists a minimal basis $B^{\prime}$ of $x$ that can be reached from $B$ in at most 
$\left|\mathcal{I}_{0}(B)\right|-1$ degenerate pivots. Moreover, if $k$ is the number of pivots required to reach $B^{\prime},\left|\mathcal{I}_{0}\left(B^{\prime}\right)\right|=\left|\mathcal{I}_{0}(B)\right|-k$.

Proof The proof is constructive. If $B$ is minimal, then trivially $B^{\prime}=B$ and $k=0$. Suppose that $B$ is not minimal. To gain minimality, the basic step consists in performing a degenerate pivot that merges two trees of the current basic subgraph through a 0 valued edge. Let $u v$ be a (basic) 0 -valued edge connecting two trees $C_{i}$ and $C_{j}$ of $G_{B}$. Note that $i, j \in \mathcal{I}_{0}(B)$ and $x_{u}+x_{v}=1$ imply either $x_{u}=1$ and $x_{v}=0$ or $x_{u}=0$ and $x_{v}=1$. We can perform the degenerate pivot defined by a ROOT-OUT operation on $\tau\left(C_{i}\right)$ and an EDGE-IN operation on $u v$, in order to merge $C_{i}$ and $C_{j}$ into a unique tree rooted at $\tau\left(C_{j}\right)$. Clearly, in the new basic subgraph the number of trees decreases by one and, as the variable leaving the basis is 0 -valued, the pivot is degenerate. We can iterate this step until, after $k$ pivots, we reach a minimal basis $B^{\prime}$ of $x$. Note that $k=\left|E\left(G_{B^{\prime}}\right) \backslash E\left(G_{B}\right)\right|$, and $\left|\mathcal{I}_{0}\left(B^{\prime}\right)\right|=\left|\mathcal{I}_{0}(B)\right|-k$. As $I(x) \neq \emptyset$ we have $\left|\mathcal{I}_{0}\left(B^{\prime}\right)\right| \geq 1$ and it follows that $k \leq\left|\mathcal{I}_{0}(B)\right|-1$. In fact, if we perform $\left|\mathcal{I}_{0}(B)\right|-1$ degenerate pivots, we obtain a 1-pseudoforest that contains only one rooted tree, i.e. the basic subgraph of a minimal basis.

Figure 5(i) shows a non-minimal basis whose basic subgraph consists of five trees. The basis is not minimal because two tree components are linked by some 0 valued edges. Unrooting one of the two trees and merging them through an EDGE-IN

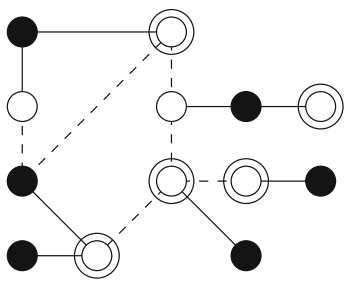

(i)

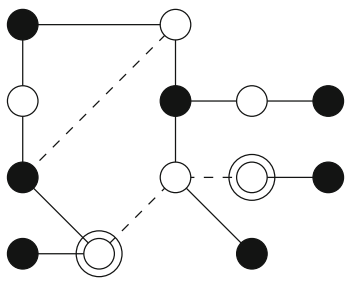

(iv)

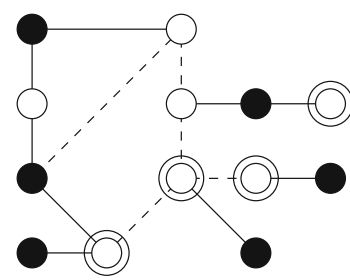

(ii)

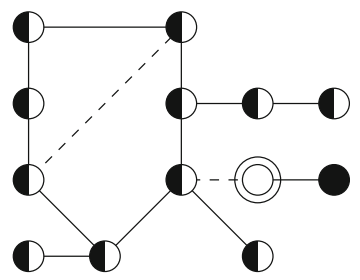

(v)

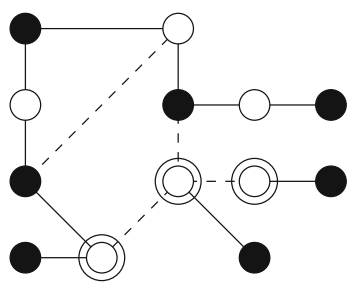

(iii)

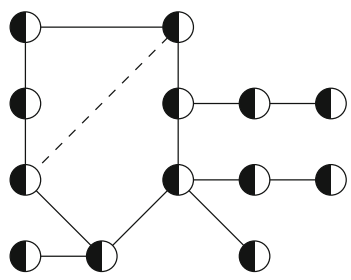

(vi)

Fig. 5 It is possible to reach $x_{i}^{*}=\frac{1}{2} \forall i \in V$ from the integer solution represented in $(i)$ in 5 pivots. The first step consists in a degenerate pivot, in order to reach a minimal basis ( $i i)$. The second step is a nondegenerate pivot leading to a new integer vertex that saturates an odd cycle of the graph ( $\mathrm{iii}$ ). The basis associated to the new vertex is not minimal, and a degenerate pivot is required, in order to regain minimality (iv). In the new minimal basis all the edges of a saturated 7-cycle are nonbasic. Therefore, in one pivot we can move to a fractional vertex $(v)$. Finally, in the last pivot, we reach $x^{*}(v i)$. In each pivot the number of rooted trees has decreased by one, therefore 5 pivots are necessary. However, the length of the path to $x^{*}$ is 3 , because we have performed two degenerate pivots 
operation on a 0 -valued edge is a degenerate pivot. The new basis, that is represented in Figure 5( $\mathrm{ii})$, is minimal and consists of four trees.

We next present a necessary and sufficient condition for a given integer vertex to be adjacent to a fractional one.

Definition 6 A cycle $\kappa$ is saturated by a stable set $S$ if it contains at most two consecutive nodes that do not belong to $S$. Equivalently, $\kappa$ contains at most one 1 -valued edge.

With a slight abuse of terminology, we say that a cycle is saturated by an integer vertex $x$ of $\operatorname{FSTAB}(G)$ if it is saturated by the corresponding stable set $S=\left\{u \in V: x_{u}=1\right\}$.

Lemma 4 Let $x$ be an integer vertex of $\operatorname{FSTAB}(G)$ and $B$ be a minimal basis associated to $x$. If $G$ admits an odd cycle $\kappa$ saturated by $x$, then it admits an odd cycle $\kappa^{\prime}$ saturated by $x$, with the property that all the 0 -valued edges of $\kappa^{\prime}$ are nonbasic.

Proof First, note that since $x$ is integer $G_{B}$ is a rooted forest, i.e. it has no 1tree component. Let $w z$ be the only 1-valued edge of the saturated odd cycle $\kappa$ and let $\rho(\kappa)$ be the set of 0 -valued edges of $\kappa$ that do not belong to $G_{B}$, i.e. $\rho(\kappa)=\left\{u v \in E: x_{u}+x_{v}=1, u v \in \kappa \cap B\right\}$. Assume, without loss of generality, that $\kappa$ is a saturated odd cycle of $G$ with minimum $|\rho(\kappa)|$. If $\rho(\kappa)$ is empty the theorem follows $\left(\kappa^{\prime} \equiv \kappa\right)$.

By contradiction, assume $\rho(\kappa) \neq \varnothing$ and let $u v \in \rho(\kappa)$. By minimality of $B$, it follows that $u, v \in V\left(C_{i}\right)$ for some tree $C_{i}$ of $G_{B}$. Let $P^{u v}$ be the path connecting $u$ and $v$ on $C_{i}$. $P^{u v}$ has odd length because $x_{u}=1-x_{v}$ and each edge of $P^{u v}$ links a 1 -valued and a 0 -valued node. Let $Q^{u v}=\kappa \backslash u v$. Note that $Q^{u v}$ has even length, and that $w z \in Q^{u v} \backslash P^{u v}$. Consider the symmetric difference $P^{u v} \Delta Q^{u v}$.

If $P^{u v} \cap Q^{u v}=\emptyset$, then $\kappa^{\prime}=P^{u v} \cup Q^{u v}$ is a saturated cycle of odd length such that $u v \notin \kappa^{\prime}$, thus $\left|\rho\left(\kappa^{\prime}\right)\right| \leq|\rho(\kappa)|-1$, a contradiction.

If $P^{u v} \cap Q^{u v} \neq \varnothing$, then $P^{u v} \Delta Q^{u v}$ is a collection of two or more cycles. Let $\kappa^{\prime \prime}$ be the cycle of $P^{u v} \Delta Q^{u v}$ containing $w z$. If the path $\kappa^{\prime \prime} \cap Q^{u v}$ has odd length, then its terminal nodes have the same value, and consequently $\kappa^{\prime \prime} \cap P^{u v}$ has even length. Otherwise, if the path $\kappa^{\prime \prime} \cap Q^{u v}$ has even length, then its terminal nodes have different values, implying that $\kappa^{\prime \prime} \cap P^{u v}$ has odd length. This shows that $\kappa^{\prime \prime}$ is an odd cycle. Moreover, $\kappa^{\prime \prime}$ is saturated by $x$, since the only two consecutive nodes of $\kappa^{\prime \prime}$ that are 0 -valued are $w$ and $z$. In addition, since $u v \notin P^{u v} \cup Q^{u v}$, then $u v \notin \kappa^{\prime \prime}$, and it follows that $\left|\rho\left(\kappa^{\prime \prime}\right)\right| \leq|\rho(\kappa)|-1$, a contradiction.

The condition of Lemma 4 is illustrated in Fig. 5(iv). An integral vertex and an associated minimal basis are represented. In the corresponding basic subgraph, there exists a saturated 5-cycle containing a 0 -valued basic edge. Nevertheless, a saturated 7 -cycle exists, such that all its 0 -valued edges are nonbasic.

Lemma 5 Let $x$ be the incidence vector of a stable set $S$ of $G$. Then $x$ is adjacent to a fractional vertex of $\operatorname{FSTAB}(G)$ if and only if there exists an odd cycle of $G$ saturated by $S$.

Proof Suppose that there exists an odd cycle of $G$ saturated by $S$. Consider a minimal basis $B$ of $x$. Note that, by Lemma 3, such basis always exists. Then, by Lemma 4, there 
exists an odd cycle $\kappa$ saturated by $x$, and such that all 0 -valued edges of $\kappa$ are nonbasic, implying that they belong to the same tree component $C_{i}$ of $G_{B}$. Denote by $w z$ the only 1 -valued edge of $\kappa$. Performing a ROOT-OUT operation on $\tau\left(C_{i}\right)$ and an EDGE-IN operation on $w z$ corresponds to a nondegenerate pivot relaxing integrality, because all nodes of $C_{i}$ become $\frac{1}{2}$-valued in the new basic solution. Moreover, minimality of $B$ implies that the new basic solution is feasible, i.e. it is a vertex: no infeasibility can occur, because each edge $u v \in E$ with $u \in V\left(C_{i}\right), v \notin V\left(C_{i}\right)$ and $x_{u}=0$ is such that $x_{v} \leq \frac{1}{2}$.

Conversely, suppose that $x$ is adjacent to a fractional vertex $x^{\prime}$. Then, by Lemma 2, there exists a spanning tree $T$ of $G\left[H\left(x^{\prime}\right)\right]$, such that for each $u v \in E(T), u v \in \delta(S)$. Recalling that $G\left[H\left(x^{\prime}\right)\right]$ is nonbipartite, it follows that there are two nodes $w, z \in$ $H\left(x^{\prime}\right) \backslash S$ such that $w z \in E$. Therefore $T \cup w z$ contains an odd cycle with only two consecutive nodes in $V \backslash S$.

The condition of Lemma 5 is illustrated in Fig. 5. In Fig. 5(i) an integral vertex is represented, such that no odd cycle of the graph is saturated by the vertex. Such vertex does not admit any fractional neighbor. Figure 5(iv) shows another integral vertex, that saturates a 7-cycle of the graph. From this vertex, in one pivot, it's in fact possible to reach an adjacent fractional vertex (Fig. 5(v)).

Theorem 5 Let $G=(V, E)$ be a nonbipartite graph. Let $x$ be a vertex of $\operatorname{FSTAB}(G)$ and $x^{*}$ be the full-fractional solution (defined by $x_{u}^{*}=\frac{1}{2} \forall u \in V$ ). Then there exists a sequence $B_{0}, B_{1}, \ldots, B_{p}$ of adjacent bases such that:

(i) the basic solutions $x^{k}=B_{k}^{-1} \mathbf{1}, k=0, \ldots, p$, are all feasible and $I\left(x^{0}\right) \supseteq$ $I\left(x^{1}\right) \supseteq \ldots \supseteq I\left(x^{p}\right)=\emptyset$;

(ii) $B_{0}$ is a basis of $x$ and $B_{p}$ is a basis of $x^{*}$;

(iii) $p$ is the number of nonbasic nodes of $G_{B_{0}}$.

Proof Let $x^{k}$ be a vertex of $\operatorname{FSTAB}(G)$ and $B_{k}$ be a minimal basis of $x^{k}$ such that $G_{B_{k}}$ has $p^{k} \geq 0$ rooted trees. If $p^{k}=0$, then $x^{k}=x^{*}$. We have the following claims. Claim (1). If $x^{k}$ is fractional and $p^{k} \geq 1$, then there exists a minimal basis $B_{k+1}$ adjacent to $B_{k}$ and such that: $x^{k+1} \in \operatorname{FSTAB}(G) ; I\left(x^{k+1}\right) \subset I\left(x^{k}\right) ; G_{B_{k+1}}$ has $p^{k}-1$ rooted trees.

$G$ is connected and hence there exists a (basic) $\frac{1}{2}$-valued edge $w z$ linking a 1-tree $C_{i}$ to a tree $C_{j}$ of $G_{B_{k}}$. We can perform a ROOT-OUT operation on $\tau\left(C_{j}\right)$ and an EDGE-IN operation on $w z$. This corresponds to a nondegenerate pivot from $B_{k}$ to $B_{k+1}$ such that $x_{u}^{k+1}=\frac{1}{2}$ for all $u \in V\left(C_{j}\right)$ and $x_{u}^{k+1}=x_{u}^{k}$ for all $u \notin V\left(C_{j}\right)$, hence $I\left(x^{k+1}\right) \subset I\left(x^{k}\right)$. By minimality of $B_{k}, B_{k+1}$ is also minimal. Moreover, $x^{k+1}$ is feasible: for each $u v \in E$ with $u \in V\left(C_{j}\right)$ and $x_{u}^{k}=0$, either $v \in V\left(C_{j}\right)$ and $x_{u}^{k+1}=x_{v}^{k+1}=\frac{1}{2}$, or $v \notin V\left(C_{j}\right)$, implying, by minimality of $B_{k}, x_{v}^{k+1}=x_{v}^{k} \leq \frac{1}{2}$ and $x_{u}^{k+1}+x_{v}^{k+1} \leq 1$. Moreover, by merging $C_{i}$ and $C_{j}$, we decrease the number of rooted trees in $G_{B_{k+1}}$. End of Claim (1).

Claim (2). If $x^{k}$ is integer and $G$ admits an odd cycle saturated by $x^{k}$, then there exists a minimal basis $B_{k+1}$ adjacent to $B_{k}$ and such that: $x^{k+1} \in \operatorname{FSTAB}(G) ; I\left(x^{k+1}\right) \subset$ $I\left(x^{k}\right)$, i.e. $x^{k+1}$ is fractional; $G_{B_{k+1}}$ has $p^{k}-1$ rooted trees. 
Consider the minimal basis $B_{k}$ associated to $x^{k}$. By Lemma $5, x^{k}$ is adjacent to a fractional solution. More precisely, by Lemma $4, x^{k}$ admits a saturated odd cycle $\kappa$ with all 0-valued edges belonging to a tree $C_{i}$ of $G_{B_{k}}$. Consider the only 1-valued edge of $\kappa$ and denote it by $w z$. The elementary transformation defined by a ROOT-OUT operation on $\tau\left(C_{i}\right)$ and an EDGE-IN operation on $w z$ is a pivot from $B_{k}$ to $B_{k+1}$, such that $x_{u}^{k+1}=\frac{1}{2}$ for all $u \in V\left(C_{i}\right)$ and $x_{u}^{k+1}=x_{u}^{k}$ for all $u \notin V\left(C_{i}\right)$, hence $I\left(x^{k+1}\right) \subset I\left(x^{k}\right)$. Moreover, $x^{k+1}$ is feasible: for each $u v \in E$ with $u \in V\left(C_{i}\right)$ and $x_{u}^{k}=0$, either $v \in V\left(C_{i}\right)$ and $x_{u}^{k+1}=x_{v}^{k+1}=\frac{1}{2}$, or $v \notin V\left(C_{i}\right)$, implying, by minimality of $B_{k}, x_{v}^{k+1}=x_{v}^{k}=0$ and $x_{u}^{k+1}+x_{v}^{k+1} \leq 1$. This proves that the new basis $B_{k+1}$ is a feasible basis, meaning that $x^{k+1}$ is a vertex of $\operatorname{FSTAB}(G)$. By construction $B_{k+1}$ is minimal and $G_{B_{k+1}}$ has $p^{k}-1$ rooted trees. End of Claim (2).

Claim (3). If $x^{k}$ is integer and no odd cycle of $G$ is saturated by $x^{k}$, then there exists a sequence of adjacent bases $B_{k}, \ldots, B_{k+q}, q \geq 1$, such that for each $j=1, \ldots, q$ : $x^{k+j} \in \operatorname{FSTAB}(G) ; x^{k+j} \neq x^{k} ; I\left(x^{k+j}\right)=I\left(x^{k}\right)$, i.e. $x^{k+j}$ is integer; $G_{B_{k+j}}$ has $p^{k}-j$ rooted trees; $B_{k+q}$ is minimal.

Consider the minimal basis $B_{k}$ associated to $x^{k}$. First, note that $p^{k} \geq 2$. In fact, if $p^{k}=1, G_{B_{k}}$ would be itself a tree and, since $G$ is nonbipartite, it would contain a saturated odd cycle. Let $C_{i}$ and $C_{j}$ be two rooted trees of $G_{B_{k}}$ that are linked by a 1 -valued edge $w z$. We can perform a ROOT-OUT operation on $\tau\left(C_{j}\right)$ and an EDGE-IN operation on $w z$. This results in a nondegenerate pivot from $B_{k}$ to $B_{k+1}$ such that $x_{u}^{k+1}=1-x_{u}^{k}$ for all $u \in V\left(C_{j}\right)$ and $x_{u}^{k+1}=x_{u}^{k}$ for all $u \notin V\left(C_{j}\right)$. By minimality of $B_{k}$, for each $u v \in E$ with $u \in V\left(C_{j}\right), v \notin V\left(C_{j}\right)$ and $x_{u}^{k}=0$, we have $x_{v}^{k+1}=x_{v}^{k}=0$, thus $x_{u}^{k+1}+x_{v}^{k+1}=1$. Moreover, because no odd cycle is saturated by $x^{k}$, we have that $G\left[V\left(C_{j}\right)\right]$ is bipartite. Therefore, for each $u v \in E$ with $u, v \in V\left(C_{j}\right)$ and $x_{u}^{k}=0$, we have $x_{v}^{k}=1$. This implies $x_{u}^{k+1}+x_{v}^{k+1}=\left(1-x_{u}^{k}\right)+\left(1-x_{v}^{k}\right)=1$. Thus $B_{k+1}$ is feasible, i.e. $x^{k+1}$ is a vertex of $\operatorname{FSTAB}(G)$, and $x^{k+1} \neq x^{k}$. By construction, $I\left(x^{k+1}\right)=I\left(x^{k}\right)$ and $G_{B_{k+1}}$ has $p^{k}-1$ rooted trees. If $B_{k+1}$ is minimal, $q=1$. Otherwise, by Lemma 3, we can reach in $h \geq 1$ degenerate pivots a minimal basis $B_{k+1+h}$ such that $x^{k+1+h}=x^{k+1}$ and $G_{B_{k+1+h}}$ has $p^{k}-1-h$ rooted trees. In this case, $q=1+h$. End of Claim (3).

Let $B$ be a basis of $x$ and $p=\left|\mathcal{I}_{0}(B)\right|$. We set $B_{0}=B$. If $B$ is not minimal, by Lemma 3 , there exists a sequence of $d$ degenerate pivots leading to a minimal basis $B_{d}$ of $x^{d}=x$, whose basic subgraph $G_{B_{d}}$ has $p-d$ rooted trees (Fig. 5(i)-(ii)). As such $d$ pivots are degenerate, $x^{1}=\cdots=x^{d}=x$, implying that the sequence $\left\{x^{0}, \cdots, x^{d}\right\}$ trivially satisfies $(i)$. If $B$ is minimal, then $d=0$.

Consider now $B_{d}$, with $d \geq 0$, and the associated vertex $x^{d}=x$.

Suppose that $x$ is integer and that there is no odd cycle of $G$ saturated by $x$. We can apply Claim (3) $t \geq 1$ times to generate $t$ sequences of adjacent bases $\left\{B_{d+j}\right\}_{j=q^{i}, q^{i}+1, \ldots, q^{i+1}, i}=0, \ldots, t-1$, with $q^{0}=0$ and $q^{i+1}>q^{i}$. The sequence $x^{d}, \ldots, x^{d+q^{t}}$ satisfies ( $i$ ): by Claim (3), for each $i=0, \ldots, t-1$ and $q^{i}<j \leq q^{i+1}, B_{d+j}$ is feasible, $x^{d+j} \neq x^{d+q^{i}}, I\left(x^{d+j}\right)=I\left(x^{d+q^{i}}\right), G_{B_{d+j}}$ has $p-d-j$ rooted trees, and $B_{d+q^{i+1}}$ is minimal. Moreover, for $q^{t} \leq p-d-1, x^{d+q^{t}}$ is an integer vertex of $\operatorname{FSTAB}(G)$ that saturates an odd cycle of $G$ (Fig. 5(ii)-(iv)). In fact, if $q^{t}=p-d-1$, then $G_{B_{d+q^{t}}}$ has only 1 rooted tree and, as $G$ is nonbipartite, 
$x^{d+q^{t}}$ saturates an odd cycle of $G$. If $x$ is integer and $G$ contains an odd cycle saturated by $x$ then, trivially, $t=0$.

Consider now $B_{d+q^{t}}$ and $x^{d+q^{t}}$, with $d, q^{t} \geq 0$. By construction, $G$ has an odd cycle saturated by the integer vertex $x^{d+q^{t}}$. By Claim (2), we can reach an adjacent basis $B_{d+q^{t}+1}$ that is minimal, feasible, such that $G_{B_{d+q^{t}+1}}$ has $p-d-q^{t}-1$ rooted trees and with $I\left(x^{d+q^{t}}\right) \supset I\left(x^{d+q^{t}+1}\right)$. This implies that $B_{d+q^{t}+1}$ satisfies $(i)$ and that $x^{d+q^{t}+1}$ is fractional (Fig. 5(iv)-(v)). Let $b \in\{0,1\}$ be such that $b=1$ if $x$ is integer and $b=0$ if $x$ is fractional. Note that, if $x$ is fractional, $q^{t}=b=0$.

Consider now $B_{d+q^{t}+b}$ and $x^{d+q^{t}+b}$, with $d, q^{t} \geq 0$ and $b \in\{0,1\}$. By construction, $x^{d+q^{t}+b}$ is a fractional vertex of $\operatorname{FSTAB}(G)$ and $B_{d+q^{t}+b}$ is minimal. Moreover, $G_{B_{d+q^{t}+b}}$ has $p-d-q^{t}-b$ rooted trees. Then, we can apply Claim (1) $p-d-q^{t}-b$ times, and generate a sequence of feasible bases $B_{d+q^{t}+b}, \ldots, B_{p}$ satisfying $(i)$. This proves that the entire sequence of bases $B_{0}, \ldots, B_{p}$ satisfies $(i)$. Furthermore, $G_{B_{p}}$ contains no rooted trees and therefore, by Theorem $3, x^{p}=x^{*}$ and $(i i)$ is also proven (Fig. 5(v)-(vi)). Finally, as $p$ is defined as the number of rooted trees of $G_{B_{0}}, p$ equals the number of nonbasic nodes of $G_{B_{0}}$. This proves (iii).

Corollary 1 Let $G$ be a nonbipartite graph and let $x$ be a vertex of $\operatorname{FSTAB}(G)$. Denote by $x^{*}$ the full-fractional vertex. There exists a path from $x$ to $x^{*}$ along the edges of $\operatorname{FSTAB}(G)$ of length at most $p$, where $p$ is the number of nonbasic nodes in a basic subgraph $G_{B}$ of $x$.

\section{The diameter of the fractional stable set polytope}

The Hirsch Conjecture (1957) states that a $d$-dimensional polytope with $f$ facets cannot have combinatorial diameter greater than $f-d$, i.e. any two vertices of the polytope are connected through a path of at most $f-d$ edges. For the fractional stable set polytope $\operatorname{FSTAB}(G)$, under Assumption 2.1, the Hirsch bound equals $|E(G)|$. We first show that, under Assumption 2.1, the diameter of $\operatorname{FSTAB}(G)$ is at most $|V(G)|$. Note that, in the nonbipartite case, $|V(G)| \leq|E(G)|$ and the Hirsch bound trivially holds. This observation, together with the result of Naddef on the diameter of $(0,1)$ polytopes and Remark 1, allows us to show that the Hirsch bound is valid for the fractional stable set polytope of any undirected simple graph.

Let $G=(V, E)$ be a graph satisfying Assumption 2.1. We prove that, from an arbitrary vertex $x$ of $\operatorname{FSTAB}(G)$, it is possible to reach any other vertex $x^{\prime}$ through a sequence of adjacent vertices $\left\{x^{t}\right\}_{t=0, \ldots, T}$, such that $x^{0}=x, x^{T}=x^{\prime}$ and $T \leq n=$ $|V|$. Our proof is constructive: each step defines a transition from the current vertex to an adjacent one. A transition may include several degerate pivots, and it always includes one nondegenerate pivot.

We partition the node set $V$ into subsets $V^{i j}=\left\{u \in V: x_{u}=i, x_{u}^{\prime}=j\right\}$, with $i, j \in\left\{0, \frac{1}{2}, 1\right\}$. Note that, if $u \in V^{i j}$ and $v \in V^{h k}$ such that $i+h>1$ or $j+k>1$, then $u v \notin E$. Moreover, $V^{01}, V^{10}, V^{\frac{1}{2} 1}, V^{1 \frac{1}{2}}$ and $V^{11}$ are stable sets, because the nodes of each subset are 1-valued in $x$ or in $x^{\prime}$. In Fig. 6 we represent the edges that can potentially occur in $G$, according to the partition of $V$ into subsets $V^{i j}, i, j \in\left\{0, \frac{1}{2}, 1\right\}$ : each edge $\left(V^{i j}, V^{h k}\right)$ expresses the possibility that a node $u \in V^{i j}$ and a node $v \in V^{h k}$ 


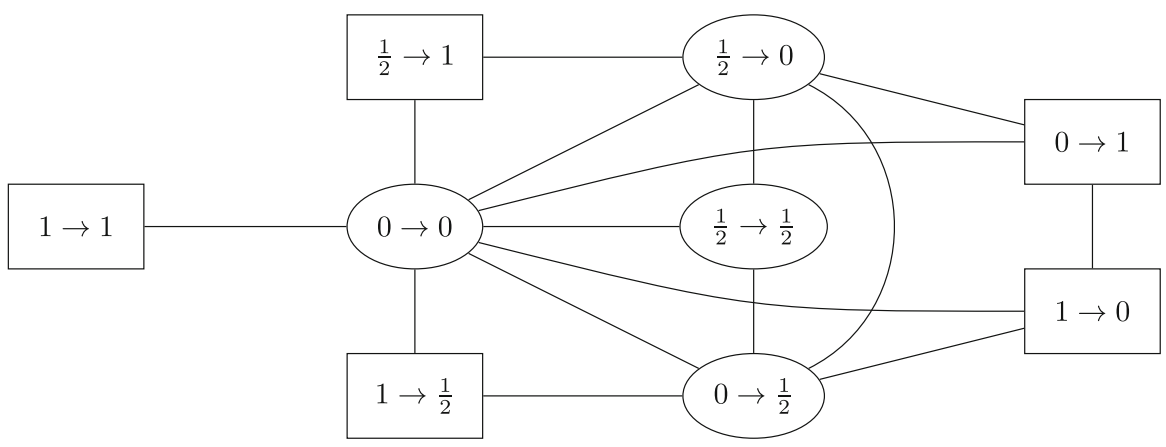

Fig. 6 The edges that can potentially occur in $G$, according to the partition of $V$ into subsets $V^{i j}, i, j \in$ $\left\{0, \frac{1}{2}, 1\right\}$. Each subset $V^{i j}$ is labeled as ' $i \rightarrow j$ '. Subsets into squares are stable, while subsets into circles need not be

are linked in $G$. If two subsets $V^{i j}$ and $V^{h k}$ are not connected by an edge, then no edge exists in $G$, that links two nodes $u \in V^{i j}, v \in V^{h k}$. The subsets represented into squares are stable, while those into circles need not be.

Recall that, for each vertex $x^{t}$ of the sequence $\left\{x^{t}\right\}$, we have defined the notation $V^{\prime}(t)=\left\{u \in V: x_{u}^{t}=x_{u}^{\prime}\right\}$. Our sequence will be such that $V^{\prime}(t+1) \supseteq V^{\prime}(t)$ for all $t=0, \ldots, T-1$ and $V^{\prime}(T)=V$, i.e. $x^{T}=x^{\prime}$. Note that $V^{\prime}(0)=V^{00} \cup V^{11} \cup V^{\frac{1}{2}} \frac{1}{2}$.

Each vector $x^{t}$ of our sequence must be a vertex, i.e. a basic feasible solution associated to some basis $B_{t}$. To achieve this, each transition from a vertex $x^{t}$ to $x^{t+1}$ corresponds to a block of pivots such that only one among them is nondegenerate. This guarantees that $x^{t+1}$ is a basic solution adjacent to $x^{t}$. To guarantee that $x^{t+1}$ is feasible, i.e. that it is a vertex of $\operatorname{FSTAB}(G)$, we also need to show that $x_{u}^{t+1}+x_{v}^{t+1} \leq 1$ for each $u v \in E$.

Let us first introduce some intermediate results.

Lemma 6 Let $x$ be a vertex of $\operatorname{FSTAB}(G)$. Then each node indexing a $\frac{1}{2}$-valued component of $x$ is connected in $G$ to another node indexing a $\frac{1}{2}$-valued component of $x$.

Proof By contradiction, assume that there is a node $v \in V$ such that $x_{v}=\frac{1}{2}$ and each edge $u v \in E$ is such that $x_{u}=0$. Then, in any basic subgraph associated to a basis of $x, v$ does not belong to any 1-tree component, which contradicts the hypothesis that $x$ is a basic feasible solution of $\operatorname{FSTAB}(G)$.

Lemma 7 Let $x \in \operatorname{FSTAB}(G)$ and $U \subset V$. Suppose that $G[U]$ does not contain isolated nodes and that $x_{u}=0$ for all $u \in N(U)$. Then, $x$ is a vertex of $\operatorname{FSTAB}(G)$ if and only if $x_{U}$ is a vertex of $\operatorname{FSTAB}(G[U])$ and $x_{V \backslash U}$ is a vertex of $\operatorname{FSTAB}(G[V \backslash U])$.

Proof Suppose that $x$ is a vertex of $\operatorname{FSTAB}(G)$. We show that there always exists a basis $B$ of $x$ such that $\delta(U) \subseteq E_{B}$, i.e. all the edges in the cutset $\delta(U)$ don't belong to $G_{B}$. Indeed, in every basic subgraph of $x$, each 1-tree is such that all its nodes belong either to $U$ or to $V \backslash U$. In fact, if there exists a 1-tree with an edge $u v \in \delta(U)$, then $x_{v}=x_{u}=\frac{1}{2}$, contradicting the hypothesis that every node in $N(U)$ is 0 -valued. Thus, we only need to prove that there exists a basic subgraph of $x$ such that each rooted tree 
has no edge in $\delta(U)$. To this purpose, it is sufficient to show that $G[U]$ and $G[V \backslash U]$ do not contain any isolated node that is 1 -valued in $x$. If this is the case, we can define two rooted forests, spanning the $(0,1)$-valued nodes of $U$ and $V \backslash U$, respectively, as follows: we link each 1-valued node of $U$ (resp. of $V \backslash U$ ) to an adjacent 0 -valued node of $U$ (resp. of $V \backslash U$ ), and we set the remaining 0-valued nodes of $U$ (resp. of $V \backslash U)$ as singletons of the rooted forest. By hypothesis, we know that $G[U]$ does not contain isolated nodes. Suppose by contradiction that $G[V \backslash U]$ contains a 1-valued isolated node $w$. Recall that, by Assumption 2.1, $G$ is a connected graph with at least an edge. It follows that $w$ has a neighbor in $U$, that contradicts $x_{w}=0$. We have shown the existence of a basis $B$ associated to $x$ such that $G_{B}$ has no edge in $\delta(U)$. This directly implies that $G_{B}[U]$ and $G_{B}[V \backslash U]$ define 1-pseudoforests of $G[U]$ and $G[V \backslash U]$, respectively, proving that $x_{U}$ is a vertex of $\operatorname{FSTAB}(G[U])$ and $x_{V \backslash U}$ is a vertex of $\operatorname{FSTAB}(G[V \backslash U])$.

To prove the converse, consider any two basic subgraphs $G_{B_{1}}$ and $G_{B_{2}}$ associated to bases $B_{1}$ and $B_{2}$ of $x_{U}$ and $x_{V \backslash U}$, respectively. Clearly, $G_{B}=G_{B_{1}} \cup G_{B_{2}}$ is a 1-pseudoforest of $G$ associated to a basis $B$ of $x$. Moreover, by hypothesis, $x_{u}=0$ for all $u \in N(U)$, implying that no constraint indexed by an edge in $\delta(U)$ is violated. This proves that $B$ is feasible, i.e. that $x$ is a vertex of $\operatorname{FSTAB}(G)$.

Lemma 8 Let $x^{1}$ be a vertex of $\operatorname{FSTAB}(G)$ and $U \subset V$ be such that $G[U]$ does not contain isolated nodes and $x_{u}^{1}=0$ for all $u \in N(U)$. Let $x^{2}$ be such that $x_{V \backslash U}^{2}=x_{V \backslash U}^{1}$. If $x_{U}^{2}$ is a vertex of $\operatorname{FSTAB}(G[U])$, then $x^{2}$ is a vertex of $\operatorname{FSTAB}(G)$. Moreover, if $x_{U}^{1}$ and $x_{U}^{2}$ are adjacent on $\operatorname{FSTAB}(G[U])$, then $x^{1}$ and $x^{2}$ are adjacent on $\operatorname{FSTAB}(G)$.

Proof By Lemma 7, $x_{V \backslash U}^{1}$ is a vertex of $\operatorname{FSTAB}(G[V \backslash U])$. As, by hypothesis, $x_{U}^{2}$ is a vertex of $\operatorname{FSTAB}(G[U])$, by Lemma 7 it follows that $x^{2}$ is a vertex of $\operatorname{FSTAB}(G)$.

Suppose now that $x_{U}^{1}$ and $x_{U}^{2}$ are adjacent vertices of $\operatorname{FSTAB}(G[U])$, i.e. that there exist two adjacent bases $B_{1}$ and $B_{2}$ of $\operatorname{FSTAB}(G[U])$ associated to $x_{U}^{1}$ and $x_{U}^{2}$, respectively. Let $B_{1}^{\prime}$ be a basis of $\operatorname{FSTAB}(G[V \backslash U])$ associated to $x_{V \backslash U}^{1}$. It follows that $B_{1}=B_{1} \cup B_{1}^{\prime}$ and $B_{2}=B_{2} \cup B_{1}^{\prime}$ are adjacent bases of $\operatorname{FSTAB}(G)$ associated to $x^{1}$ and $x^{2}$, respectively.

Theorem 6 The combinatorial diameter of $\operatorname{FSTAB}(G)$ is at most $|V(G)|$.

Proof Let $n=|V(G)|$. Let $x$ and $x^{\prime}$ be two vertices of $\operatorname{FSTAB}(G)$. Our goal is to define a sequence of adjacent vertices $\left\{x^{t}\right\}_{t=0, \ldots, T}$, such that $x^{0}=x, x^{T}=x^{\prime}$ and $T \leq n$. We will perform four blocks of transitions and, correspondingly, we will define four vertices $x^{t^{1}}, x^{t^{2}}, x^{t^{3}}, x^{t^{4}}$ with $t^{1} \leq t^{2} \leq t^{3} \leq t^{4}=T$. Each transition consists in a sequence of pivots, such that exactly one among these pivots is nondegenerate. We will show that the four blocks of transitions are such that: $V^{\prime}\left(t^{1}\right) \backslash V^{\prime}(0)=V^{10}$ and $t^{1}=$ $\left|V^{10}\right|, V^{\prime}\left(t^{2}\right) \backslash V^{\prime}\left(t^{1}\right)=V^{1 \frac{1}{2}} \cup V^{0 \frac{1}{2}}$ and $t^{2}-t^{1} \leq\left|V^{0 \frac{1}{2}}\right|, V^{\prime}\left(t^{3}\right) \backslash V^{\prime}\left(t^{2}\right)=V^{\frac{1}{2} 1} \cup V^{\frac{1}{2} 0}$ and $t^{3}-t^{2} \leq\left|V^{\frac{1}{2} 0}\right|, V^{\prime}\left(t^{4}\right) \backslash V^{\prime}\left(t^{3}\right)=V^{01}$ and $t^{4}-t^{3}=\left|V^{01}\right|$. As a consequence

$$
\begin{aligned}
T & =t^{4}=\left(t^{4}-t^{3}\right)+\left(t^{3}-t^{2}\right)+\left(t^{2}-t^{1}\right)+t^{1} \\
& \leq\left|V^{01}\right|+\left|V^{\frac{1}{2} 0}\right|+\left|V^{0 \frac{1}{2}}\right|+\left|V^{10}\right| \leq|V| .
\end{aligned}
$$

We remark that, if $G$ is bipartite, $V^{\frac{1}{2} 0}=V^{0 \frac{1}{2}}=V^{\frac{1}{2} 1}=V^{1 \frac{1}{2}}=V^{\frac{1}{2} \frac{1}{2}}=\emptyset$. 
In the first block of transitions we set to zero, one by one, nodes of $V^{10}$, i.e. for $t=$ $0, \ldots, t^{1}-1$ and $u \in V^{10} \backslash V^{\prime}(t)$, we set $x_{u}^{t+1}=0$ and $x_{v}^{t+1}=x_{v}^{t}$ for all $v \neq u$. Clearly, no edge constraint (1) can be violated by $x^{t+1}$, therefore $x^{t+1}$ is feasible. To show that each vector of the sequence is a basic solution, we associate to it a 1-pseudoforest, that univocally defines a basis. Let $B \in \mathcal{B}$ be a basis associated to $x=x^{0}$ and let $G_{B}$ be the corresponding basic subgraph. Suppose that in $G_{B}$ a node $u \in V^{10}$ has degree greater than one and let $C_{i}, i \in \mathcal{I}_{0}(B)$, be the tree such that $u \in V\left(C_{i}\right)$. Let $u v \in E\left(G_{B}\right)$ be an edge of $C_{i}$ that does not belong to the path between $u$ and $\tau\left(C_{i}\right)$ on $C_{i}$. This implies $v \in V_{B}$. Because nonbasic edges are 0 -valued, we have $x_{v}=0$. Then we can perform the degenerate pivot defined by an EDGE-OUT operation on $u v$ and a ROOT-IN operation on $v$, to define a new 1-pseudoforest where the degree of $u$ has decreased by one. Therefore, we can always define a 1-pseudoforest of $x$ where each $u \in V^{10}$ has degree one. Assume w.l.o.g. that $B$ defines a 1-pseudoforest $G_{B}$ satisfying this condition.

For $t=0, \ldots, t^{1}-1$, let $B_{t}$ be a basis associated to $x^{t}$, and suppose that in $G_{B_{t}}$ each node of $V^{10} \backslash V^{\prime}(t)$ has degree one. Let $u \in V^{10} \backslash V^{\prime}(t)$ and $u v$ be the only nonbasic edge incident on $u$. Then, we can perform an EDGE-OUT operation on $u v$ and a ROOT-IN operation on $u$, that corresponds to a nondegenerate pivot yielding a new basis $B_{t+1}$ and a basic feasible solution $x^{t+1}$. In the new 1-pseudoforest $G_{B_{t+1}}$, every node in $V^{10} \backslash V^{\prime}(t+1)$ has degree one and $V^{\prime}(t+1)=V^{\prime}(t) \cup\{u\}$. In other words, $x_{u}^{t+1}=0$ and $x_{v}^{t+1}=x_{v}^{t}$ for all $v \neq u$. We have shown that $x^{t+1}$ is a vertex adjacent to $x^{t}$ for $t=0, \ldots, t^{1}-1$. Vertex $x^{t^{1}}$ is defined, for each $v \in V$, as:

$$
x_{v}^{t^{1}}= \begin{cases}0 & v \in V^{10}, \\ x_{v} & v \in V \backslash V^{10}\end{cases}
$$

(see Fig. 7). This shows that $x^{0}, \ldots, x^{t^{1}}$ is a sequence of adjacent vertices of $\operatorname{FSTAB}(G)$ of length $\left|V^{10}\right|$ and such that $V^{\prime}\left(t^{1}\right)=V^{\prime}(0) \cup V^{10}$.

In the second block of transitions we change the values of nodes of $V^{1 \frac{1}{2}} \cup V^{0 \frac{1}{2}}$. Let $U=H(x) \cup H\left(x^{\prime}\right)$. Our goal is to define a sequence $x^{t^{1}}, \ldots, x^{t^{2}}$ of adjacent vertices such that, for each $v \in V$

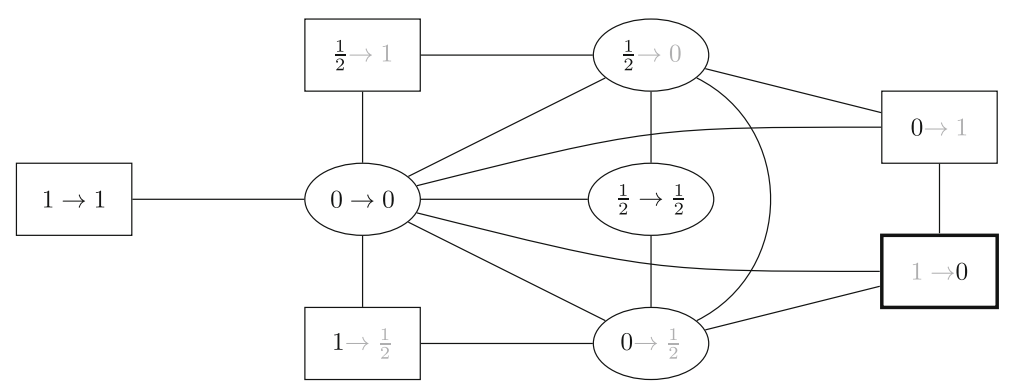

Fig. 7 The first block of transitions yields vertex $x^{t^{1}}$. For each subset $V^{i j}$ with $i \neq j$, in the label ' $i \rightarrow j$ ' either $i$ or $j$ is highlighted, depending on the value of the corresponding variables in $x^{t^{1}}$ 


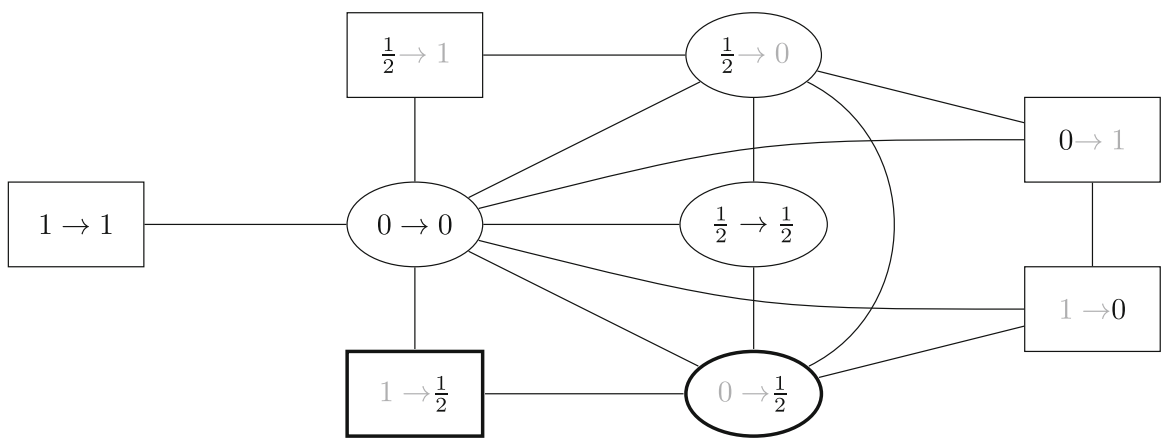

Fig. 8 The second block of transitions yields vertex $x^{t^{2}}$. For each subset $V^{i j}$ with $i \neq j$, in the label ' $i \rightarrow j$ ' either $i$ or $j$ is highlighted, depending on the value of the corresponding variables in $x^{2}$

$$
x_{v}^{t^{2}}= \begin{cases}\frac{1}{2} & v \in U, \\ x_{v}^{t^{1}} & v \in V \backslash U,\end{cases}
$$

i.e. $V^{\prime}\left(t^{2}\right)=V^{\prime}\left(t^{1}\right) \cup V^{1 \frac{1}{2}} \cup V^{0 \frac{1}{2}}$ and $H\left(x^{t^{2}}\right)=U$ (see Fig. 8). If $V^{0 \frac{1}{2}} \cup V^{1 \frac{1}{2}}=\emptyset$, then $t^{2}=t^{1}$. Suppose $V^{1 \frac{1}{2}} \cup V^{0 \frac{1}{2}} \neq \emptyset$. By Lemma 6 , the induced subgraph $G[U]$ does not contain isolated nodes. Moreover, for each $v \in N(U)$ we have that $x_{v}^{t^{1}}=0$ (see Fig. 7). Thus, by Lemma $7, x_{U}^{t^{1}}$ and $x_{V \backslash U}^{t^{1}}$ are vertices of $\operatorname{FSTAB}(G[U])$ and $\operatorname{FSTAB}(G[V \backslash U])$, respectively. By Corollary 1 , we can generate a sequence $\left\{x_{U}^{t}\right\}_{t=t^{1}, \ldots, t^{2}}$ of adjacent vertices of $\operatorname{FSTAB}(G[U])$, where $x^{t^{2}}$ is full-fractional on $U$, and $t^{2}-t^{1}$ is at most the number of nonbasic nodes in a basic subgraph associated to $x_{U}^{t^{1}}$, implying $t^{2}-t^{1} \leq\left|V^{0 \frac{1}{2}}\right|$.

By Lemma 8, for each $t^{1}<t \leq t^{2}$, we can lift vertex $x_{U}^{t}$ of $\operatorname{FSTAB}(G[U])$ to a vertex $x^{t}$ of $\operatorname{FSTAB}(G)$ by setting

$$
x^{t}=\left(\begin{array}{c}
x_{U}^{t} \\
x_{V \backslash U}^{t^{1}}
\end{array}\right) .
$$

Lemma 8 also implies that $x^{t}$ and $x^{t+1}$ are adjacent for all $t^{1} \leq t<t^{2}$. This shows that $x^{0}, \ldots, x^{t^{1}}, \ldots, x^{t^{2}}$ is a sequence of adjacent vertices of $\operatorname{FSTAB}(G)$ of length at $\operatorname{most}\left|V^{10}\right|+\left|V^{0 \frac{1}{2}}\right|$ and such that $V^{\prime}\left(t^{2}\right)=V^{\prime}(0) \cup V^{10} \cup V^{0 \frac{1}{2}} \cup V^{1 \frac{1}{2}}$.

In the third block of transitions we change the values of nodes of $V^{\frac{1}{2} 1} \cup V^{\frac{1}{2} 0}$. Our goal is to define a sequence $x^{t^{2}}$, dots, $x^{t^{3}}$ of adjacent vertices such that, for each $v \in V$

$$
x_{v}^{t^{3}}= \begin{cases}x_{v}^{\prime} & v \in U, \\ x_{v}^{t^{1}} & v \in V \backslash U,\end{cases}
$$

i.e. $V^{\prime}\left(t^{3}\right)=V^{\prime}\left(t^{2}\right) \cup V^{\frac{1}{2} 1} \cup V^{\frac{1}{2} 0}$ (see Fig. 9). If $V^{\frac{1}{2} 1} \cup V^{\frac{1}{2} 0}=\emptyset$, then $t^{3}=t^{2}$. In particular, if $G$ is bipartite, $t^{3}=t^{1}$. Suppose $V^{\frac{1}{2} 1} \cup V^{\frac{1}{2} 0} \neq \emptyset$. 


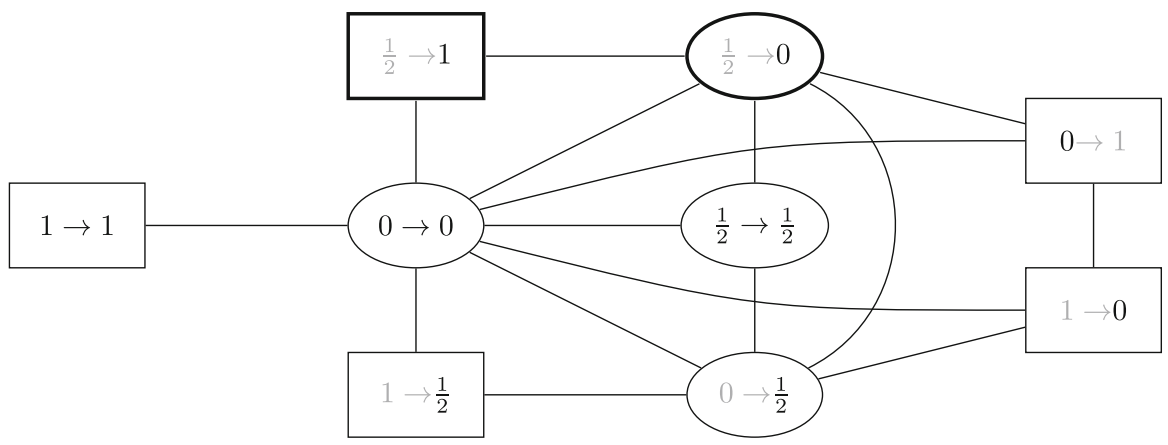

Fig. 9 The third block of transitions yields vertex $x^{t^{3}}$. For each subset $V^{i j}$ with $i \neq j$, in the label ' $i \rightarrow j$ ' either $i$ or $j$ is highlighted, depending on the value of the corresponding variables in $x^{t^{3}}$

We first show that $x_{U}^{\prime}$ is a vertex of $\operatorname{FSTAB}(G[U])$. Let $B^{\prime}$ be a basis of $x^{\prime}$. Then $G_{B^{\prime}}\left[H\left(x^{\prime}\right)\right]$ is a collection of 1 -trees spanning the nodes of $V^{1 \frac{1}{2}} \cup V^{0 \frac{1}{2}} \cup V^{\frac{1}{2}} \frac{1}{2}$. Moreover, by applying Lemma 6 to $x$, it follows that in $G$ each node of $V^{\frac{1}{2}} 1$ is linked to a node in $V^{\frac{1}{2} 0}$. Therefore, we can define a rooted forest $F$ that spans the nodes of $V^{\frac{1}{2} 1} \cup V^{\frac{1}{2} 0}$ as follows: we link each node of $V^{\frac{1}{2} 1}$ to a node in $V^{\frac{1}{2} 0}$, and we set the remaining nodes of $V^{\frac{1}{2} 0}$ as singletons of $F$. We then set all the nodes in $V^{\frac{1}{2} 0}$ as the roots of $F$. It follows that $G_{B^{\prime}}\left[H\left(x^{\prime}\right)\right] \cup F$ is a 1-pseudoforest associated to $x_{U}^{\prime}$, proving that $x_{U}^{\prime}$ is a vertex of $\operatorname{FSTAB}(G[U])$.

As $x^{t^{2}}$ is full-fractional on $U$, by Corollary 1 there exists a path between $x_{U}^{\prime}$ and $x_{U}^{t^{2}}$ along edges of $\operatorname{FSTAB}(G[U])$, whose length is bounded by the number of nonbasic nodes in a basic subgraph associated to $x_{U}^{\prime}$. As this number cannot exceed $\left|V^{\frac{1}{2} 0}\right|$, we can generate a sequence $\left\{x_{U}^{t}\right\}_{t=t^{2}, \ldots, t^{3}}$ of adjacent vertices of $\operatorname{FSTAB}(G[U])$, where $x_{U}^{t^{3}}=x_{U}^{\prime}$ and $t^{3}-t^{2} \leq\left|V^{\frac{1}{2} 0}\right|$.

Recall that $x_{V \backslash U}^{t^{1}}$ is a vertex of FSTAB $(G[V \backslash U])$. Then, by Lemma 8, for each $t^{2}<$ $t \leq t^{3}$, we can lift vertex $x_{U}^{t}$ of $\operatorname{FSTAB}(G[U])$ to a vertex $x^{t}$ of $\operatorname{FSTAB}(G)$ by setting

$$
x^{t}=\left(\begin{array}{c}
x_{U}^{t} \\
x_{V \backslash U}^{t^{1}}
\end{array}\right)
$$

Lemma 8 also implies that $x^{t}$ and $x^{t+1}$ are adjacent for all $t^{2} \leq t<t^{3}$. This shows that $x^{0}, \ldots, x^{t^{1}}, \ldots, x^{t^{2}}, \ldots, x^{t^{3}}$ is a sequence of adjacent vertices of $\operatorname{FSTAB}(G)$ of length at most $\left|V^{10}\right|+\left|V^{0 \frac{1}{2}}\right|+\left|V^{\frac{1}{2} 0}\right|$ and such that $V^{\prime}\left(t^{3}\right)=V^{\prime}(0) \cup V^{10} \cup V^{0 \frac{1}{2}} \cup$ $V^{1 \frac{1}{2}} \cup V^{\frac{1}{2} 0} \cup V^{\frac{1}{2} 1}$.

Finally, in the fourth block of transitions we set to 1 , one by one, nodes of $V^{01}$, i.e. for $t=t^{3}, \ldots, t^{4}-1$ and $u \in V^{01} \backslash V^{\prime}(t)$, we set $x_{u}^{t+1}=1$ and $x_{v}^{t+1}=x_{v}^{t}$ for all $v \neq u$. No edge constraint (1) can be violated by $x^{t+1}$, because for each $u \in V^{01}$ and $u v \in E, v \in$ $V^{00} \cup V^{\frac{1}{2} 0} \cup V^{10}$, therefore $x_{v}^{t+1}=x_{v}^{t^{3}}=0$. To show that each vector of the sequence is a basic solution, we associate to it a 1-pseudoforest, that univocally defines a basis. 


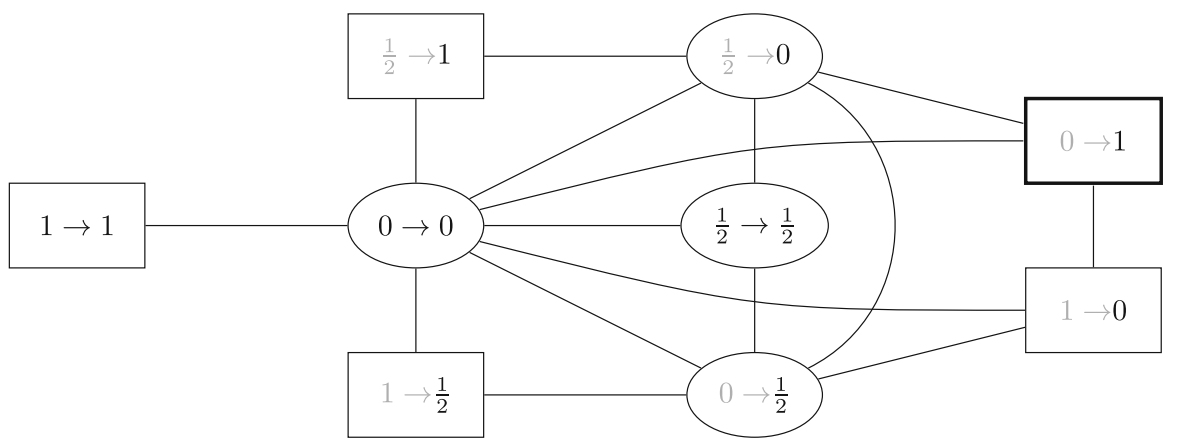

Fig. 10 The fourth block of transitions yields vertex $x^{t^{4}}=x^{\prime}$. For each subset $V^{i j}$ with $i \neq j$, in the label ' $i \rightarrow j$ ' either $i$ or $j$ is highlighted, depending on the value of the corresponding variables in $x^{4}$.

For $t=t^{3}, \ldots, t^{4}-1$, suppose that $x^{t}$ is a vertex of $\operatorname{FSTAB}(G)$, and let $B_{t}$ be a basis associated to $x^{t}$. In $G_{B_{t}}$ each node $u \in V^{01} \backslash V^{\prime}(t)$ is a singleton, i.e. a trivial rooted tree, because all edges in $\delta(u)$ are 1-valued, therefore basic (see Fig. 9). On the other hand, by Assumption 2.1, $|\delta(u)| \geq 1$. Let $u v \in E$ be an edge incident on $u$ in $G$. We have $x_{u}^{t}=x_{v}^{t}=0$. We can perform a ROOT-OUT operation on $u$ and and an EDGEIN operation on $u v$, i.e. a nondegenerate pivot yielding a new basis $B_{t+1}$ and a basic feasible solution $x^{t+1}$. We have $V^{\prime}(t+1)=V^{\prime}(t) \cup\{u\}$, i.e. $x_{u}^{t+1}=1$ and $x_{v}^{t+1}=x_{v}^{t}$ for all $v \neq u$. We have shown that $x^{t+1}$ is a vertex adjacent to $x^{t}$ for $t=t^{3}, \ldots, t^{4}-1$. Moreover, $t^{4}-t^{3}=\left|V^{01}\right|$ and $V^{\prime}\left(t^{4}\right)=V$, i.e. $x^{t^{4}}=x^{\prime}$. (see Fig. 10). This shows that $x=x^{0}, \ldots, x^{t^{1}}, \ldots, x^{t^{2}}, \ldots, x^{t^{3}}, \ldots, x^{t^{4}}=x^{\prime}$ is a sequence of adjacent vertices of $\operatorname{FSTAB}(G)$ of length at most $\left|V^{10}\right|+\left|V^{0 \frac{1}{2}}\right|+\left|V^{\frac{1}{2} 0}\right|+\left|V^{01}\right| \leq|V|$. An example of the procedure to build the sequence $\left\{x^{t}\right\}_{t=0, \ldots, T}$ that we have described above is presented in Fig. 11.

We can now discard Assumption 2.1, and state a more general result.

Corollary 2 Let $G=(V, E)$ be a simple, undirected graph and denote by $Z$ the subset of isolated nodes of $G$. The combinatorial diameter of $\operatorname{FSTAB}(G)$ is at most $|Z|+\min \{|V \backslash Z|,|E|\}$.

Proof Suppose that $G$ consists of $k$ connected components $G^{1}, \ldots, G^{k}$. By Remark 1 , $d(\operatorname{FSTAB}(G))=\sum_{i=1}^{k} d\left(\operatorname{FSTAB}\left(G^{i}\right)\right)$. If $G^{i}$ is an isolated node, then $d\left(\operatorname{FSTAB}\left(G^{i}\right)\right)$ $=1$. If $G^{i}$ is bipartite and $\left|V\left(G^{i}\right)\right| \geq 2$, then $\operatorname{FSTAB}\left(G^{i}\right)=\operatorname{STAB}\left(G^{i}\right)$ and $d\left(\operatorname{FSTAB}\left(G^{i}\right)\right) \leq \min \left\{\left|V\left(G^{i}\right)\right|,\left|E\left(G^{i}\right)\right|\right\}[12]$. If $G^{i}$ is nonbipartite, by Theorem $6, d\left(\operatorname{FSTAB}\left(G^{i}\right)\right) \leq\left|V\left(G^{i}\right)\right| \leq\left|E\left(G^{i}\right)\right|$. Therefore

$$
\begin{aligned}
d(\operatorname{FSTAB}(G)) & =\sum_{i=1}^{k} d\left(\operatorname{FSTAB}\left(G^{i}\right)\right) \leq|Z|+\sum_{i:\left|V\left(G^{i}\right)\right|>1} \min \left\{\left|V\left(G^{i}\right)\right|,\left|E\left(G^{i}\right)\right|\right\} \\
& \leq|Z|+\min \{|V \backslash Z|,|E|\} .
\end{aligned}
$$




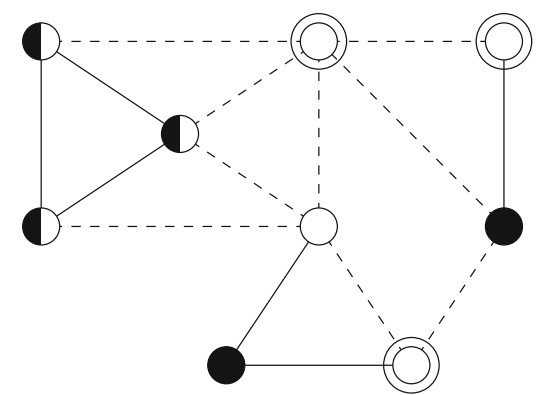

$x=x^{0}$

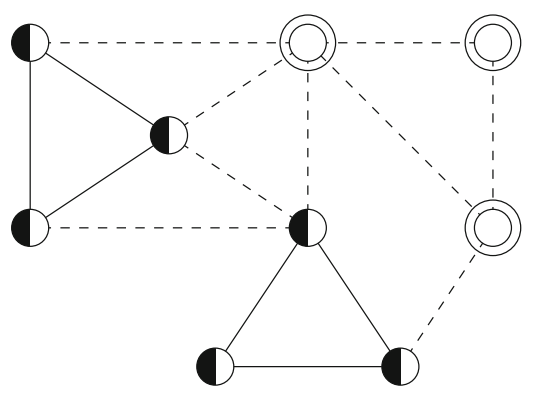

$x^{t^{2}}, t^{2}=2$

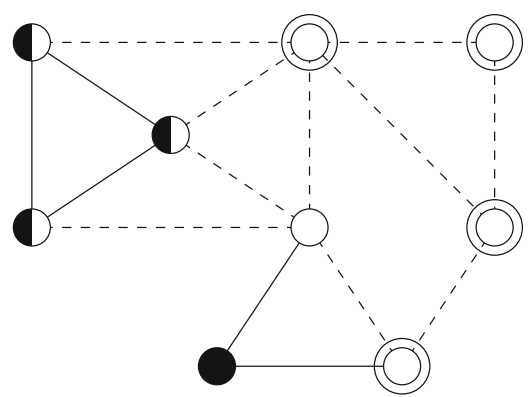

$x^{t^{1}}, t^{1}=1$

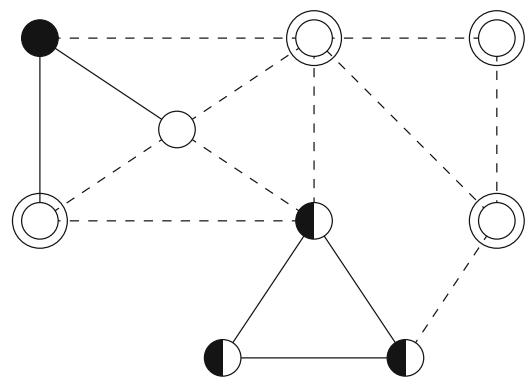

$x^{t^{3}}, t^{3}=3$

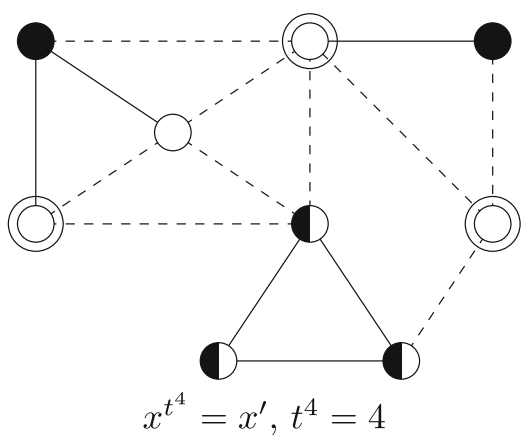

Fig. 11 A graph $G$ with 9 nodes and 14 edges. Given two vertices $x$ and $x^{\prime}$, a sequence $x=$ $x^{0}, x^{1}, x^{2}, x^{3}, x^{4}=x^{\prime}$ of adjacent vertices of $\operatorname{FSTAB}(G)$ is constructed, according to the constructive proof of Theorem 6 . In this example we can reach $x^{\prime}$ from $x$ in four pivots, with one pivot per block of transitions.

Corollary 3 Let $G=(V, E)$ be a simple, undirected graph. The combinatorial diameter of $\operatorname{FSTAB}(G)$ is at most $|V|$.

Corollary 4 Let $G=(V, E)$ be a simple, undirected graph and denote by $Z$ the subset of isolated nodes of $G$. The combinatorial diameter of $\operatorname{FSTAB}(G)$ is at most $|E|+|Z|$, i.e. the Hirsch bound is valid for $\operatorname{FSTAB}(G)$. 
Acknowledgments We thank the referees for their valuable comments, that greatly helped to improve the presentation of the paper.

\section{References}

1. Balinski, M.: On Maximum Matching, Minimum Covering and Their Connections. In: H. Kuhn (ed.) Proceedings of the Princeton symposium on, mathematical programming, pp. 303-312 (1970)

2. Balinski, M.: The Hirsch conjecture for dual transportation polyhedra. Math. Oper. Res. 9(4), 629-633 (1984)

3. Balinski, M., Russakoff, A.: On the assignment polytope. Siam Review 16 (1974)

4. Balinski, M., Rispoli, F.J.: Signature classes of transportation polytopes. Math. Program. 60(1-3), 127-144 (1993)

5. Brightwell, G., van den Heuvel, J., Stougie, L.: A linear bound on the diameter of the transportation polytope. Combinatorica 26(2), 133-139 (2006)

6. Campêlo, M., Cornuéjols, G.: Stable sets, corner polyhedra and the Chvátal closure. Oper. Res. Lett. 37(6), 375-378 (2009)

7. Cornuéjols, G., Michini, C., Nannicini, G.: How tight is the corner relaxation? Insights gained from the stable set problem. Discret. Optimiz. 9(2), 109-121 (2012)

8. Dantzig, G.: Linear Programming and Extensions. A Rand Corporation Research Study. Princeton University Press (1998)

9. Ikura, Y., Nemhauser, G.L.: An efficient primal simplex algorithm for maximum weighted vertex packing on bipartite graphs. In: Bachem, A., Grötschel, M., Korte, B. (eds.) Bonn Workshop on Combinatorial Optimization, North-Holland Mathematics Studies, vol. 66, pp. 149-168. North-Holland (1982)

10. Klee, V., Walkup, D.: The $d$-step conjecture for polyhedra of dimension $d<6$. Acta. Math. 117, 53-78 (1967)

11. Michini, C.: The stable set problem: some structural properties and relaxations. Ph.D. thesis, Sapienza Università di Roma (2012)

12. Naddef, D.: The Hirsch Conjecture is true for (0, 1)-polytopes. Math. Program. 45, 109-110 (1989)

13. Nemhauser, G.L., Trotter, L.E.: Vertex packings: structural properties and algorithms. Math. Program. 8, 232-248 (1975)

14. Padberg, M.W.: On the facial structure of set packing polyhedra. Math. Program. 5, 199-215 (1973)

15. Santos, F.: A counter example to the Hirsch Conjecture. Ann. Math. 176, 383-412 (2012)

16. Trubin, V.A.: On a method of solution of integer linear programming problems of a special kind. Soviet Math. Dokl. 10, 1544-1596 (1969) 